\title{
External Control of Electron Temperature in Ultra-cold Plasmas
}

Roy O. Wilson

Colby College

Follow this and additional works at: https://digitalcommons.colby.edu/honorstheses

Part of the Astrophysics and Astronomy Commons

Colby College theses are protected by copyright. They may be viewed or downloaded from this site for the purposes of research and scholarship. Reproduction or distribution for commercial purposes is prohibited without written permission of the author.

\section{Recommended Citation}

Wilson, Roy O., "External Control of Electron Temperature in Ultra-cold Plasmas" (2007). Honors Theses. Paper 231.

https://digitalcommons.colby.edu/honorstheses/231

This Honors Thesis (Open Access) is brought to you for free and open access by the Student Research at Digital Commons @ Colby. It has been accepted for inclusion in Honors Theses by an authorized administrator of Digital Commons@ Colby. 


\section{External Control of Electron Temperature in Ultra-cold Plasmas}

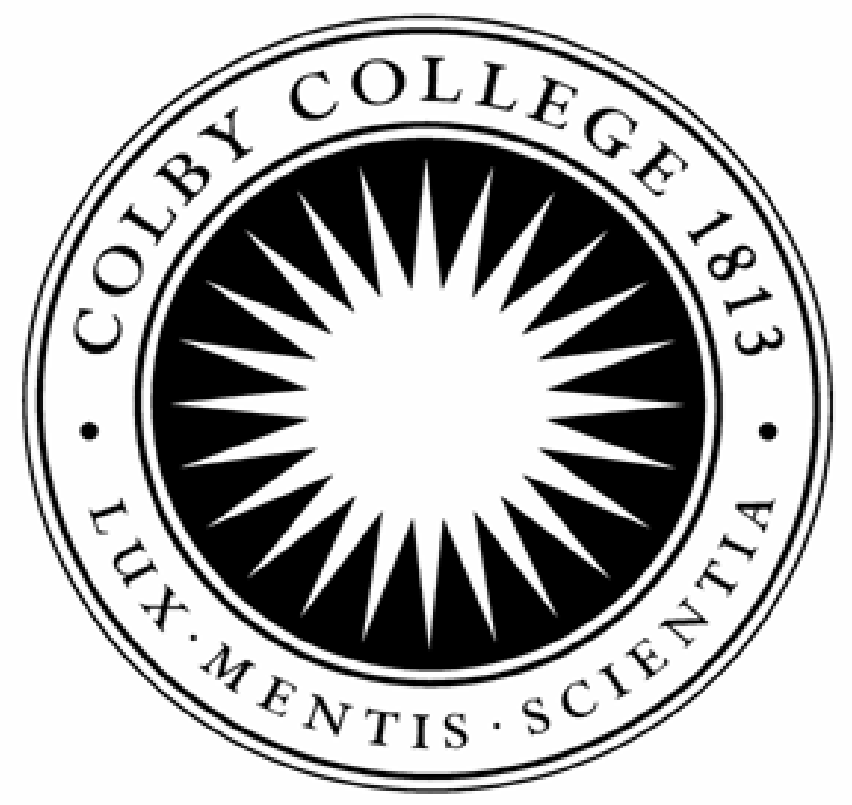

Roy O.Wilson

Honors Thesis

Colby College Department of Physics and Astronomy May 2007 


\begin{abstract}
This thesis discusses progress towards achieving external control of the electron temperature and the Coulomb coupling parameter of ultra-cold plasmas. Using a Littman dye laser, we create the plasma by partially photoionizing a dense, cold sample of rubidium atoms in a magneto-optical trap (MOT). At a controllable time delay, we excite neutral atoms in the plasma to a specific Rydberg state using a narrow bandwidth pulsed dye laser. We have made progress towards optimizing and quantifying the achievable Rydberg atom density by using mm-wave spectroscopy to control the evolution of a cold dense Rydberg sample to plasma and have also begun preliminary investigations of plasma electron temperature measurements.
\end{abstract}




\section{Acknowledgments}

Writing this thesis was no easy task. It was a project that without the guidance of some very important supporters I would not have completed. Of these supporters are the Colby College Department of Physics and Astronomy, some classmates and fellow Physics majors of the class of 2007, my family, and my very best friends.

Becoming a Physics major at Colby College is one of the best ideas I ever had. The Physics department has always been incredibly supportive of my academic endeavors and for this I am extremely grateful. I would like to extend much of my gratitude to Professor Duncan A. Tate. As my long time advisor at Colby, and my honors thesis advisor, he has expressed nothing but patience and a willingness to teach me all about ultra-cold plasmas. It was very comforting knowing I had a most excellent resource in Professor Tate throughout the completion of the project. Also, to Professor Charles W.S. Conover, a wonderful mentor and friend, I extend my thanks to as well. The first to give me a job working in the department, Professor Conover is the sole reason I became a Physics major. And a final thanks to Professor Robert T. Bluhm, Professor Murray F. Campbell, Professor Virginia C. Long, and Lisa M. Lessard. This team of Physics educators are a fabulous group, and I would recommend anyone considering Physics as a major at Colby College, to do so solely for the chance of having them as their primary instructors.

This project is the capstone of, not only the honors degree, but my Colby experience as a whole. The fellow members of my graduating class, also with Physics degrees, were very important parts of the experience. Much of my motivation to complete the project came from those guys, and I thank them as well. My family, always supporting most of my decisions, definitely supporting 
my attendance to Colby, and my decision to major in Physics, was a very strong ally. Although any mention of ultra-cold plasmas, Rydberg atoms, and atom traps in the household is met with blank stares, and an incessant need to change conversation, they remained still yet another crucial force driving my work. My deepest thanks to them as well. And to my closest friends, the most gracious and important influence on my years at Colby. Much of the sensibility and sanity I needed to complete this project derived from a priceless and stalwart support network and the most sincere of companionships. And for that, I owe you guys so much. 


\section{Contents}

Abstract

Acknowledgments ii

List of Figures vi vi vis

1 Introduction 1

1.1 Properties of Rydberg atoms . . . . . . . . . . . . . . 3

1.2 Rydberg Gas Evolution to Plasma . . . . . . . . . . . . . 3

1.3 Rydberg Atoms Embedded in Plasma . . . . . . . . . . . . 8

2 Apparatus 11

2.1 Laser Cooling and Trapping . . . . . . . . . . . . . 12

2.1.1 Optical Molasses . . . . . . . . . . . . . . . . 13

$2.1 .2 \quad$ Magnetic Traps . . . . . . . . . . . . . . . . . . . . 14

2.1.3 Magneto-Optical Trap . . . . . . . . . . . . . . . . 15

2.2 Trapping Lasers . . . . . . . . . . . . . . . . . . . . . . 18

2.2.1 External Cavity Diode Laser (ECDL) . . . . . . . . . . 19

2.2.2 Saturated Absorption Spectroscopy _. . . . . . . . . 20

2.2 .3 Injection Locking . . . . . . . . . . . . . . . . 21 
2.3 Experiments on Rydberg Atoms . . . . . . . . . . . . . . . 23

2.3.1 Pulsed Dye Lasers . . . . . . . . . . . . . . . . . . 24

2.3.2 Millimeter-wave Spectroscopy . . . . . . . . . . . . 26

2.3.3 State Selective Field Ionization (SSFI) . . . . . . . . . . . 31

2.3.4 Timing Sequence ................ . . 33

3 Experiments and Results $\quad 35$

3.1 Evolution of Rydberg Atoms to Plasma . . . . . . . . . . . . . . 35

3.2 Two-Laser Experiment . . . . . . . . . . . . . . . . . . . . 38

4 Summary and Conclusions $\quad 42$

Bibliography $\quad 44$ 


\section{List of Figures}

1.1 Ion signal versus the initial population of the $36 \mathrm{~d}$ state . . . . . . 6

1.2 Ion signal as a function of delay for a given initial population $N$ of the $\mathrm{Rb} 40 \mathrm{~d}$ state. From reference $[22]$. . . . . . . . . . . . . 7

2.1 The experimental setup for the position dependent force and velocity dependent drag force in a magneto-optical trap. From reference $[20] . \ldots \ldots \ldots \ldots \ldots \ldots$

2.2 The arrangement of the MOT in 1D . . . . . . . . . . . . 17

2.3 The cavity arrangement of an external cavity diode laser in Littrow

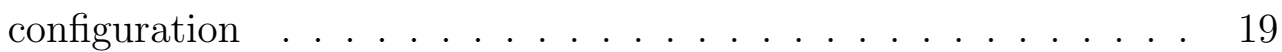

2.4 Experimental setup of a saturated absorption spectrometer. From

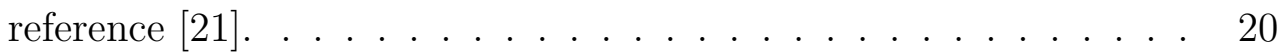

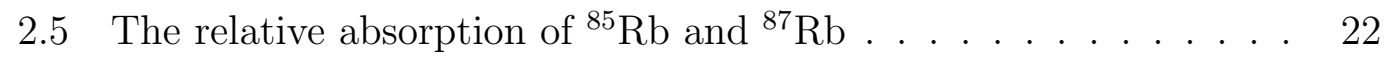

2.6 The pulsed-amplified CW laser system . . . . . . . . . . 25

2.7 Dipole interaction and associated potential energy between pairs of Rydberg atoms . . . . . . . . . . . . . . . . . . . 28

2.8 Perturbation of energy levels in neighboring ${ }^{85} \mathrm{Rb}$ atoms . . . . . . 29

2.9 Time sequence of mm-wave spectroscopy on Rydberg atoms . . . 30

2.10 Millimeter wave spectrum at different Rydberg densities . . . . . 31 
2.11 The general sequence of events during our experiments . . . . . . 32

3.1 Electron time-of-flight signal from the field ionization (FI) of $40 \mathrm{~d}_{5 / 2}$ Rb atoms and the plasma that forms . . . . . . . . . 36

3.2 Electron time of flight data showing the dependence the plasma evolution has on the Rydberg atom density . . . . . . . . . . . . . 38

3.3 Electron time-of-flight signals from an ultra cold plasma collected in our two-color experiments . . . . . . . . . . . . . . . . . . . 39 


\section{Chapter 1}

\section{Introduction}

This project is a study of the dynamics and structure of ultra-cold Rydberg gases and ultra-cold plasmas. Plasmas are electrically neutral, highly ionized gases composed of ions, electrons, and neutral particles. We are concerned with the forces between neutrals and ions, the resulting dynamics in this kind of environment, and the subsequent exchanges of energy that occur. The advent of laser cooling and trapping of atoms and the sub-kelvin temperatures obtainable by those methods is what makes our system unique from most plasmas (e.g. plasma found in fluorescent light tubes, the Sun, and fusion reactors). For most plasmas, the lowest possible temperature is that corresponding to electron energies that lead to the ionization of neutral atoms and therefore the replenishing of ions and electrons lost to the vacuum chamber walls [1]. The energies that yield ionization on impact are on the order of $5 \mathrm{eV}(55,000 \mathrm{~K})$. So plasma characterized by a temperature of 10,000 K can be considered "cold." Our ultra-cold plasma is created by photoionization of a sample of trapped ${ }^{85} \mathrm{Rb}$ atoms in a magneto-optical trap (MOT) using a pulsed dye-amplified diode laser. The initial observation of this used $50 \mu \mathrm{K}$ Xe atoms, but the results have been reproduced using cold Rb, Cs, 
and $\mathrm{Sr}$ atoms $[1,2,3]$. To a good approximation, the initial electron temperature is obtained from the remaining photon energies in excess of the ionization threshold of ${ }^{85} \mathrm{Rb}$; and the initial positive ions maintain the same temperature as the initially trapped atoms $[1,2,3]$. Thus, the ultra-cold system sustains temperatures some 10 orders of magnitude below most plasmas.

This thesis addresses three main components to this research. First, we study the evolution process from a cold Rydberg atomic gas to plasma. Second, the dynamics of slow moving Rydberg atoms interacting with a co-existing, quasineutral, cold plasma are of interest. These two threads seek to investigate the likelihood of Rydberg atoms exerting a controllable influence on the plasma temperature. We discuss their details further in the sections that follow. The third component involves long range interactions that occur within a dense, low temperature environment, and how, according to Greene et. al. [4], such interactions should permit the formation of molecular Rydberg states. Greene et. al. describe theoretically that two qualitatively different classes of molecular Rydberg states should be observable in an ultra-cold atomic gas: polar and nonpolar. Polarity here refers to the existence of permanent electric dipole moments, where the polar class exhibits permanent dipole moments and the nonpolar classes do not. This result is interesting because it predicts a homonuclear diatomic molecule to possess a permanent dipole moment that can be manipulated by electric and magnetic fields. It also suggests the possibility of absorption spectroscopy by probing this electric dipole with the electric field of electromagnetic radiation. It is these opportunities for external control that motivate this research, and that is elucidated by this thesis. 


\subsection{Properties of Rydberg atoms}

A Rydberg atom is a highly excited atom corresponding to energy states of high principal quantum number $n$. The classical picture is an electron in a large circular orbit about a positively charged ion core consisting of $Z$ protons and $Z-1$ electrons. In this orbit the electron experiences, to very good approximation, a

hydrogenic, $\frac{1}{r}$ Coulomb potential. If the outer electron penetrates the inner electron shells, it will experience a greater force as a result of seeing more positive charge. Because this effective potential can be approximated to the hydrogen potential, the valence electron wavefunction approximates to classical orbits [5]. This property explains the behavior of Rydberg electrons in low angular momentum states: that is, if the valence electron has very low angular momentum (classically interpreted as highly eccentric orbits) then it may pass close enough to the nucleus to induce a net polarization over the atom's spatial distribution, giving rise to a dipole term in the potential. Dipole-dipole forces between neighboring Rydberg atoms is also a prominent focus in this research [16]. It is also relevant to note that because of the large orbits, Rydberg electrons are loosely bound, almost "free," and therefore are susceptible to the presence of external electric fields and magnetic fields. Many experiments have been done to probe and analyze these peculiar properties. Our work and the work of those doing cold plasma research, observes the mechanisms for ionization of Rydberg atoms to plasma and the subsequent interactions between Rydberg atoms and plasma.

\subsection{Rydberg Gas Evolution to Plasma}

Before the advent of laser cooling, in the late 1970's to early 1980's, the evolution of Rydberg atoms to plasma was investigated under room temperature conditions. 
Papers released by Matsuzawa in 1979 [7] and Higgs et. al. [8] documented the results of collisional interactions within a highly excited gas. The Matsuzawa paper is a theoretical investigation that treats the Rydberg electron as if it were "free," with its interaction with neighboring neutral rare-gas atoms playing a vital role in the evolution of the system. The cross sections of $n l$-changing collisions of the Rydberg atoms with the rare-gas atoms are reported for small angular momentum changes. Higgs et. al. investigated thermal collisions between Xe (nf) Rydberg atoms and HF molecules. The products of the highly excited collisions were detected using selective field ionization and both $n$-changing and $l$-changing collisions were identified. In addition to the $n l$-mixing results, Higgs et. al. showed that ionization of Rydberg atoms is also a significant product of these collisions. Much of this work was abandoned until laser cooling and trapping was realized, and as a by-product, the creation of ultra-cold Rydberg atoms and ultra-cold plasma. The spontaneous evolution of a dense cold sample of Rydberg atoms to plasma has been demonstrated by Robinson et. al. [22] at the University of Virginia, the Laboratoire Aime Cotton in France, by Li et. al. [1], and in our labs at Colby College. Given that each atom is bound by as much as $100 \mathrm{~cm}^{-1}$, considerations of energy conservation must be raised: What mechanisms supply the energy for ionization?

The evolution from Rydberg atoms to plasma is described as follows. We excite ${ }^{85} \mathrm{Rb}$ atoms trapped in a MOT to high- $n$ Rydberg states $(n>30)$. A few microseconds after the excitation, the Rydberg atom sample begins to ionize. This ionization is due to photoionization by blackbody radiation and collisions with hot Rydberg atoms remaining in the gas. The initial electrons produced by this ionization escape the sample, and as a result a strong positive space charge builds up. After sometime this space charge becomes sufficiently strong 
to produce a Coulomb potential that traps subsequent electrons remaining in the sample. These trapped electrons then oscillate back and forth throughout the spatial region of the cloud, ionizing neutral atoms via electron-Rydberg atom collisions. This latter process is coined "avalanche ionization" and is what leads to plasma formation.

As described, the evolution of Rydberg atoms into an ultra-cold plasma is dictated by the ionizing collisions between free electrons and neutral atoms. Also, the mechanisms for the initial production of free electrons, ionizing collisions between hot and cold Rydberg atoms and blackbody photoionization, are crucial to plasma formation. The results of Robinson et. al. [22] support experimentally these proposed mechanisms. Their experiments were done with both $\mathrm{Rb}$ and Cs held in a vapor cell MOT. They analyzed the evolution via selective field ionization (SFI) and detected electrons (or ions) on a micro-channel plate (MCP) detector. The MCP signal had two components, one due to plasma ions or electrons, and one due to the initially populated 36d Rydberg state. An example is depicted in the inset of Fig. 1.1.

From the inset we that at $2 \mu$ s the sample it mostly made up of $36 \mathrm{~d}$ state atoms. At $5 \mu$ s the plasma signal is visible, and at $12 \mu$ s the Rydberg atoms have almost completely evolved to plasma. Fig. 1.1 also shows, as function of delay, the dependence of the plasma formation on the number of 36d atoms populated by the excitation laser. This data was collected using neutral density filters to vary the $36 \mathrm{~d}$ population. At a fixed delay we see that there are thresholds at which the ion signal grows rapidly, denoted by $N_{T}$ in the Fig. 1.1, and that below this threshold value of $36 \mathrm{~d}$ atoms, plasma formation does not occur. It is also relevant to note that at a high initial value of atoms the plasma signal approaches the value of $36 \mathrm{~d}$ atoms, indicating apparently complete ionization of the Rydberg 


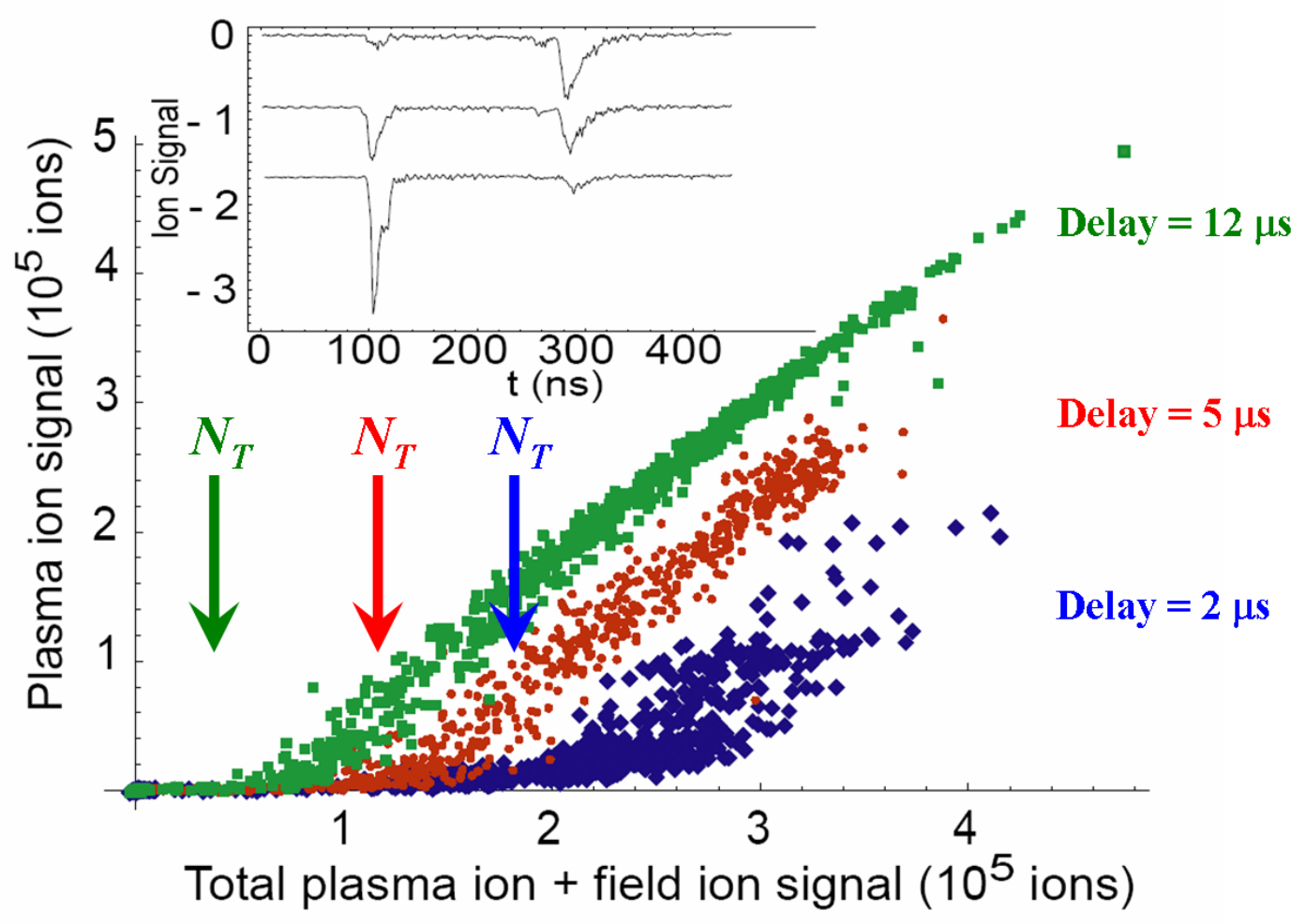

Figure 1.1: Ion signal as a function of the initial population of the Rb 36 d state. The three signals show the ion signals at $2 \mu \mathrm{s}, 5 \mu \mathrm{s}$, and $12 \mu \mathrm{s}$ delays after the excitation of the Rydberg state. The inset is the time resolved signals for an initial population of $1.9 \times 10^{5}$ atoms, featuring delays of $2 \mu \mathrm{s}, 5 \mu \mathrm{s}$, and $12 \mu \mathrm{s}$ after excitation of the 36d Rydberg state. From reference [22].

sample [22]. That there is also a threshold ion signal where avalanche ionization occurs is demonstrated by Fig. 1.2. This threshold value is independent of the number of atoms $N$. What is clear is that the time that it takes the samples to reach the threshold ion value does depend on $N$, taking longer for the lower $N$ value and less time for the higher $N$.

The following observations by Robinson et. al. support our description of the evolution from Rydberg atoms to plasma. First, they applied a small static field, $2 \mathrm{~V} / \mathrm{cm}$, and observed that the plasma never forms. This is because the plasma electrons cannot be trapped as a result of the pull experienced due to the presence 


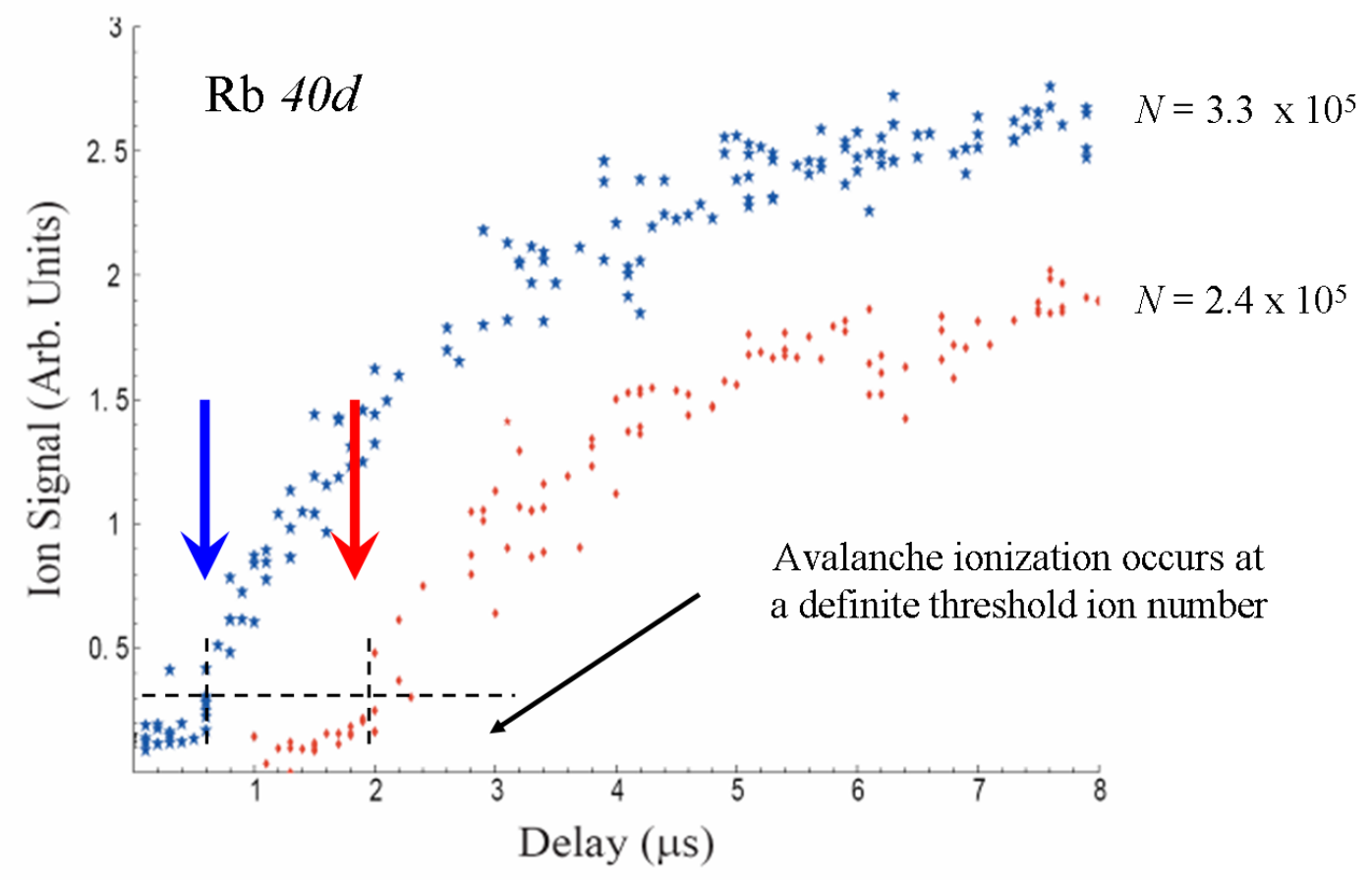

Figure 1.2: Ion signal as a function of delay for a given initial population $\mathrm{N}$ of the $\mathrm{Rb} 40 \mathrm{~d}$ state $\left(N=3.3 \times 10^{5}\right.$ and $\left.N=2.4 \times 10^{5}\right)$. There is a $1 \mu$ s offset between the two data curves for clarity. From reference [22].

of the static field. Then they observed that a sample of only hot Rydberg atoms does not lead to plasma formation. This was seen by turning off the trapping lasers and using a pulsed $780 \mathrm{~nm}$ laser to excite the $5 \mathrm{~s}_{1 / 2}$ to $5 \mathrm{p}_{3 / 2}$ trapping transition, then applying the second laser to produce hot Rydberg atoms. It is seen that without the MOT, i.e. without the presence of cold atoms, the plasma does not form, as the cloud of ions formed here expands too rapidly to trap electrons [22]. Finally, it was observed that the removal of hot atoms does not lead to the formation of a plasma for initial states of $n \leq 40$. But as for states of $n>50$ the plasma does form when hot atoms are removed. This result is reasonable in that the cold atoms are nearly stationary and therefore yield fewer ionizing collisions. For higher principal quantum numbers, $n>40$, it has been shown by Li. et. al. [23] that the attractive dipole-dipole interaction between cold Rydberg atoms 
leads to them colliding and to producing the initial electrons and ions necessary to form a plasma, a process known as Penning ionization. Robinson et. al. also made measurements of ionization rates for Cs atoms exited to 39d Rydberg states that are consistent with blackbody photoionization rates measured as a function of temperature by Spencer et. al. [24]. And this supports the suggestion that black-body photoionization plays a role in the initial ionization.

In the work done by Li. et. al. [1] the energy conservation considerations were resolved. In the initial study of Robison et. al. [22] the population seemed to localize in either the initial Rydberg state or in the plasma. One expected to see a redistribution of the final $\mathrm{nl}$-states to compensate for the energy used in producing the plasma. In later experiments, it was found that electron-Rydberg-atom collisions that occur once the trap depth is sufficient do in fact cause redistribution of the population from the initial Rydberg state to nearby high- $n l$ states. As a result of these collisions, approximately $\frac{2}{3}$ of the Rydberg atoms ionize while $\frac{1}{3}$ are de-excited to lower energy states. Li. et. al. [23] reproduce this result and attribute it to the dipole-dipole interaction of two Rydberg atoms. A pair of atoms that experience the attractive dipole force (see Fig. 2.7) will collide and produce an ion, electron, and a more deeply bound atom [23].

\subsection{Rydberg Atoms Embedded in Plasma}

The challenge undertaken by ultra-cold plasma research is the creation of strongly coupled plasma. These are plasmas in which the Coulomb interaction dominates the thermal energy of the plasma particles. Coupling is quantified by the Coulomb coupling parameter $\Gamma$, the ratio of the Coulomb interaction energy to the kinetic energies. It is represented as $\Gamma=\frac{e^{2}}{4 \pi \epsilon_{0} a k_{B} T}$, where $e$ is elementary charge, $a$ 
is the interparticle distance, $k_{B}$ is Boltzmann's constant, and $T$ is the plasma temperature. Examples of plasmas that may achieve the highly coupled regime $(\Gamma>1)$ are created from our optical atom traps (reduction of thermal energy $k_{B} T$ leads to an increase in the coupling parameter), and like those suggested by Pohl et. al. in Penning traps [9]. Pohl et. al. describe theoretically that in general the ionic component of the plasma may exhibit short-range ordering or even long range ordering because of the highly correlated state the plasma is produced in via photoionization (or indirect ionization via evolution of a Rydberg gas). This ordering is signified by differentiated patterns in the ionic plasma, evident also in crystalline and latticelike structures, and that forms as a result of high interparticle interaction energies. [9].

It has been suggested by Killian et. al. that it is possible to cool the plasma with lasers, further taking advantage of radiative pressures to impose a controllable force that limits the plasma expansion provided that the positive ions have optically accessible transitions [10]. The alternative we have embarked on is to use the binding energy of Rydberg states as "ice cubes" to cool the plasma [2]. The observations made by Vanhaecke et. al. suggest that long interaction times between Rydberg atoms and an ultra-cold plasma favor rapid ionization of the Rydberg atoms, suggesting agreement with the collisional model for avalanche ionization [2]. In the considerations of energy conservation during the evolution of Rydberg atoms to plasma the suggested use of Rydberg binding energies to cool the plasma is apparent. That is, electron collisions with very highly excited Rydberg atoms sufficiently decrease the binding energy of the Rydberg atom until it ionizes. The result is a decrease in the plasma electron temperature. On the other hand, collisions with low-lying Rydberg states gradually increase the Rydberg binding energy, resulting in a increase in the plasma electron temperature 
[2]. Our experiment investigates the possibility of controlling this mechanism. We hope that embedding Rydberg atoms of the appropriate $n$ state in an already existing plasma will result in the cooling of plasma electrons, thereby increasing the Coulomb coupling parameter $\Gamma$. 


\section{Chapter 2}

\section{Apparatus}

The main part of our apparatus is a vapor cell magneto-optical trap (MOT) of rubidium gas. The trap is contained in a stainless steel vacuum chamber pumped by a 20 liter/s ion pump with a base pressure of less than $1 \times 10^{-9}$ torr. The atoms are trapped midway between two parallel plates made from high-transparency stainless steel mesh; and with a voltage placed across the plates one can apply electric fields to the trapped sample. The chamber is in the form of a cylinder whose dimensions (i.e. diameter $150 \mathrm{~mm}$, height $200 \mathrm{~mm}$ ) allow ample access for trapping laser beams, pulsed lasers, and mm-wave radiation. There is also a 20 Gauss/cm magnetic field gradient in the center of the trap, produced by a pair of anti-Helmholtz coils on the outside of the vacuum chamber (air cooled during operation). This magnetic field gradient coupled with the radiative forces on atoms in the path of a laser beam produces the trap. A detailed description of this process and the various elements used to produce the MOT are in the sections that follow. 


\subsection{Laser Cooling and Trapping}

Laser cooling and trapping allows one to control atomic motion with extreme precision. The cooling and trapping schemes developed are highly dependent on the interaction between atoms and laser light, and it is the special properties of this interaction that allows one to exert controllable forces on the atoms. What happens in these experiments is that the speeds of the atoms interacting with the light are reduced by a considerable amount. Because the temperature is a direct measure of the distribution of velocities of a sample of atoms, we say that we are "cooling" the gas by reducing the speeds of the atoms moving in the sample. One should note that when we speak about "temperature" in laser cooling some major subtleties apply. In thermodynamics, temperature is a parameter used to describe a system that is in thermal equilibrium with its surroundings, requiring thermal contact and heat exchange. This is not the case in a laser cooling experiment because the sample of atoms is always absorbing and scattering light, making many changes with its environment. Thus the system is never in thermal equilibrium, and the assignment of a "temperature" is out of place. Nevertheless, an assignment of temperature is useful in describing a sample of atoms whose average kinetic energy $\left\langle E_{k}\right\rangle$ (and therefore its temperature) has been reduced by laser light, and we make this association as $\left\langle E_{k}\right\rangle=\frac{1}{2} k_{B} T$, where $k_{B}$ is Boltzmann's constant, and $T$ is the temperature [11].

In laser cooling, the forces on the atoms result from an exchange of momentum between the atomic sample and the laser light. Consider the interaction between an initially stationary two-level atom and a single light beam. The interaction is characterized by finite and discrete changes in momentum. In addition to the change in energy $E=\hbar \omega$, each photon imparts on the interacting atom an 
amount of momentum, $p=\hbar k[11]$. When the atom absorbs light, it stores the energy by going into an excited state; it conserves momentum by recoiling from the light source with momentum $\hbar k$. These changes in momenta are small compared to the magnitude of the atomic momenta at thermal velocities and thus the scattering of a single photon has a relatively small effect on the motion of hot atoms. But when the atoms decay from the excited state through spontaneous emission (the lifetime of the $5 \mathrm{p}_{3 / 2}$ state is $\tau=26.6 \mathrm{~ns}$ ), the associated recoil is in a random direction and the average effect over many such events causes an appreciable change in the momenta and velocities of the atom, resulting in cooling. The speeds associated with atoms at room temperature are reduced to the order of $10^{-1} \mathrm{~m} / \mathrm{s}$. It is important to note that the force that the light field exerts on the atoms does not increase without limit, but saturates to the value $\frac{\hbar k \gamma}{2}$, where $\gamma$ is the rate at which laser photons scatter from the atoms $\left(\gamma=\frac{1}{\tau}\right)$, and $\hbar k$ is the momentum per photon. The factor of $\frac{1}{2}$ comes from the force's dependence on the probability of the atom being in the excited state, which has a maximum of $\frac{1}{2}$. Note also that increasing the rate of absorption by increasing the intensity of the laser beam eventually results in stimulated emission, which does not result in cooling as the recoil is opposite that of absorption.

\subsubsection{Optical Molasses}

In a single-beam setup, the net force on atoms will have the effect of either slowing or heating, depending on the motion of the atoms relative to the propagation direction of the light field and the detuning of lasers from atomic resonance (red/blue detuned). If the atoms are moving parallel to the direction of propagation of the light then the force will speed them along if the laser is blue detuned. On the other hand, the atoms that move in the opposing direction will experience 
the effect of slowing if the laser is red detuned. With this in mind, extending the setup to two counter-propagating beams of equal frequency, and intensity allows us to remove the effect of heating provided that the laser is red detuned and we can inhibit absorptions that increase atomic momenta by appropriate choice of laser polarization. By tuning the lasers just below the resonant absorption frequency, atoms at rest will experience zero net force, but those moving along the beam path will experience a force proportional to their velocity [11]. This is a direct application of the Doppler effect. The frequency of the light that propagates in opposite direction to the atomic motion will be blue shifted in the atomic rest frame and be closer to resonance; while the beam moving in the same direction as that of the atomic motion will be red shifted, further out of resonance. Therefore, atoms will interact more strongly with the light that opposes their motion and slow down [11]. This technique is known as optical molasses. By using three orthogonal retroreflected beams, one can extend this from the one dimensional example described here to a three-dimensional one. Atoms moving along the intersection region of the beams will be restricted in three dimensions, resulting in a greater volume of atoms being cooled. It is important to note though that 3D optical molasses does not constitute a "trap," as there is no force restricting the atoms from leaving the intersection region of the beams.

\subsubsection{Magnetic Traps}

The Stern-Gerlach experiment in 1924 was the first to demonstrate the deflection of neutral atoms by an inhomogeneous magnetic field. This is a result of the atoms having magnetic moment $\mu$, and its interaction with a magnetic field. Because of the magnetic moment we can think of neutral atoms as little bar magnets. Work is done when the orientation of the dipole changes in the presence of the field, storing 
potential energy, $U=-\vec{\mu} \cdot \vec{B}$, with the associated force, $\overrightarrow{\boldsymbol{F}}=\nabla(\vec{\mu} \cdot \vec{B})$. Adequate reduction of the kinetic energies of the atoms results in the loading of a trap that exploits this force on the atoms. The key to exploiting this force is that atoms in quantum states whose energy increases with increasing field are trappable [13]. Consider the fact that atoms will be distributed over various allowed energies due to the quantization of the magnetic moment. Atoms that are aligned with the field will have lower energies at high-field and tend to occupy those regions where field maxima occur. Conversely, atoms that are anti-aligned with the field will have low energies with weak field, and therefore seek out field minima. A chargeand current-free region of space cannot sustain a field maximum, but can yield a minimum. Thus, the traps that we construct in a vacuum region will confine "weak-field seeking" particles of potential energy $U=\mu B$ [12].

We use a quadrupole trap produced by a pair of identical copper wires with oppositely directed currents, colloquially known as "anti-Helmholtz" configuration. Because the currents in the wires are in opposite directions, there is a zero field minimum at the center where the weak-field seeking atoms will coalesce. This is an experimentally attractive setup because of its simplicity of construction and optimal optical access. The quadrupole trap is commonly used because of these attributes $[2,3,4,5]$.

\subsubsection{Magneto-Optical Trap}

The efficacy of an atom trap depends on both a velocity dependent drag force and a position dependent restoring force. Optical molasses provides the velocitydependent force. The restoring force is formed by the combination of inhomogeneous magnetic fields and properly polarized light. The most widely used trap, using both optical and magnetic forces to produce a trap of neutral atoms, is 


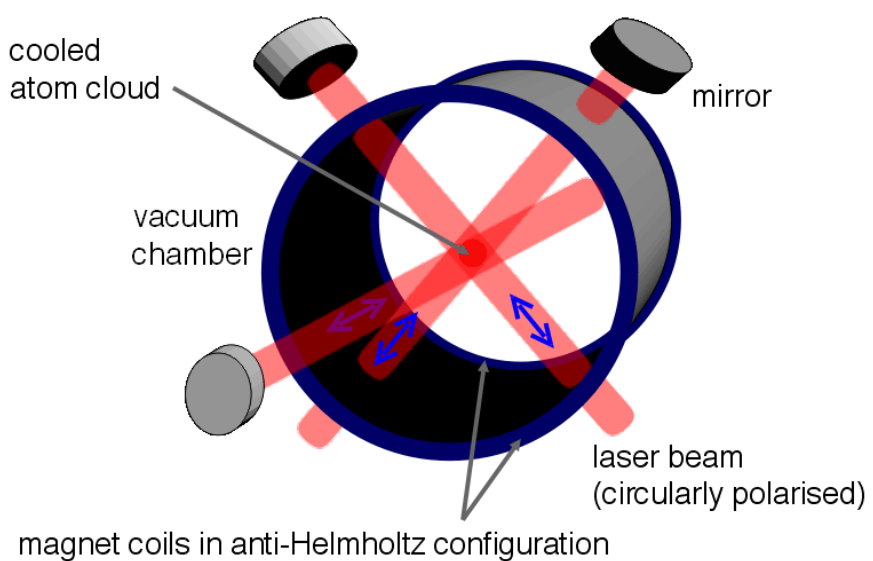

Figure 2.1: The experimental setup for the position dependent force and velocity dependent drag force in a magneto-optical trap. From reference [20].

the magneto-optical trap (see Fig. 2.1), first demonstrated by Raab et. al. [15]. The magneto-optical trap (MOT) combines these principles to form a substantial trapping potential for neutral atoms.

The counter-propagating laser has a detuning $\delta$ from the atomic transition frequency as seen by atoms at rest at the center of the trap (see Fig. 2.2). Consider the simplified case where $F=0$ is the ground state and $F=1$ is the excited state ( $F$ is stated since we are concerned with the hyperfine structure). The presence of the magnetic field raises the degeneracy of the excited state, no longer making the total atomic angular momentum quantum number, $F$, a good quantum number. The quantum number that best characterizes the energy levels in the system is the projection of total angular momentum on the $z$-axis, $M_{f}$. The $M_{f}=+1$ state is shifted up for $B>0$, and the $M_{f}=-1$ state is shifted down. The important thing is the transition selection rules that arise, and as a consequence, orbital angular momentum conservation. Each photon carries angular momentum $\hbar$, and $M_{f}$ can change by \pm 1 [11]. Conservation of orbital angular momentum requires that absorption of a photon is complemented by a corresponding change in the projection of the orbital angular momentum of an 


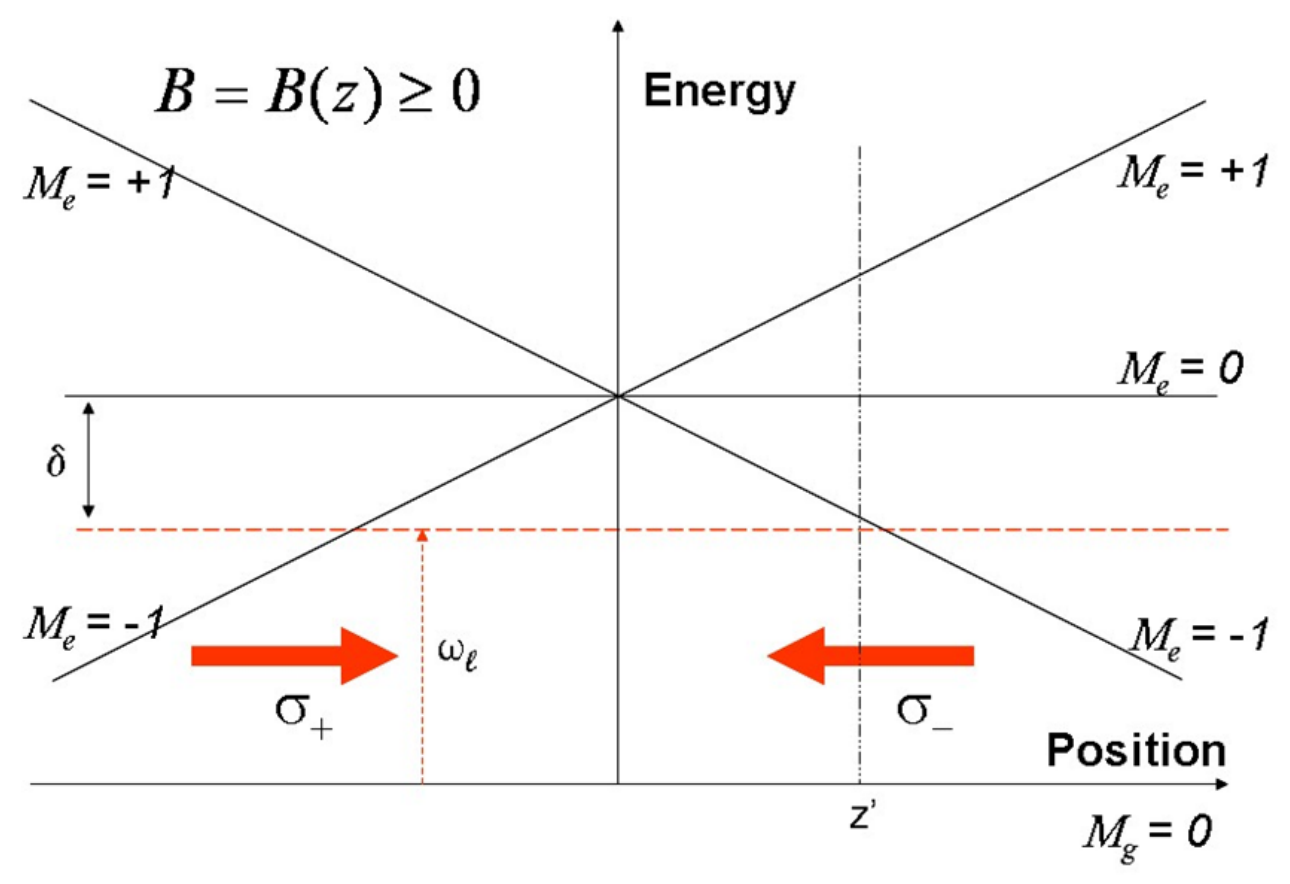

Figure 2.2: The arrangement of the MOT in 1D. The dashed line represents the detuning from the resonant frequency seen by atoms at the center of the trap. Zeeman shifts of the atomic energy are due to the presence of an inhomogeneous magnetic field. The light is circularly polarized to match the resulting selection rules, and to preserve conservation of angular momentum. From reference [11].

atom [11]. In our setup, the light is circularly polarized and therefore couples states that follow the $\Delta M_{f}= \pm 1$ selection rule [11]. Right-handed polarized light $(\sigma+)$ drives $\Delta M_{f}=+1$ transitions, and left-handed polarized light $(\sigma-)$ drives $\Delta M_{f}=-1$ transitions. The laser interacts more with atoms that opposes its direction of propagation. Atoms moving towards the $\sigma-$ source will have $\Delta M_{f}=$ -1 transition induced, and move towards the center of the trap, experiencing the molasses slowing force. Atoms moving towards the $\sigma+$ source will have the $\Delta M_{f}=+1$ induced, and also move towards the center of the trap, again experiencing the molasses slowing force. Since at the center of the trap the magnetic field has zero magnitude, the weak-field seeking atoms in the ensemble will coalesce there with potential energy $\mu B$. The reduction of kinetic energies 
loads this potential well, which subsequently keeps a hold on the atoms at the trap center. Note that the splitting of the energy levels reduces as we move towards the center of the trap, allowing for resonance over a greater range of atomic velocities as the changing Doppler shift of decelerating atoms is compensated for the changing Zeeman shift as the atoms move into the center [11]. Also note that detuning is critical because is ensures that as atoms move across the trapping region they will not be reheated by further absorptions of radiation. Furthermore, this process depends on the recycling efficiency of the transition coupled by the trapping laser, that is, we must maintain a closed system where spontaneous emission is mostly between the two states coupled by our laser. We trap ${ }^{85} \mathrm{Rb}$ using the $F=3 \rightarrow F^{\prime}=4$ transition. However, there is a nearby $\Delta F=0$ transition that will result in atoms decaying to the $F=2$ state. This requires a second laser, the repumper laser, that cycles these atoms through the $F=2 \rightarrow F^{\prime}=3$ transition. Atoms can then decay to the $F=3$ state and recouple to the trapping laser.

\subsection{Trapping Lasers}

The production of a MOT is highly dependent on our ability to control the output frequencies of our trapping lasers. We use the tuning capabilities of external cavity diode lasers in Littrow configuration to produce trapping and repumper beams. Saturated absorption spectroscopy is used to monitor and stabilize the operating frequency of the diode lasers. Also, the trap volume is dependent on the trapping laser power. We use an injection-locking amplifier system to produce laser powers sufficient for trapping. 


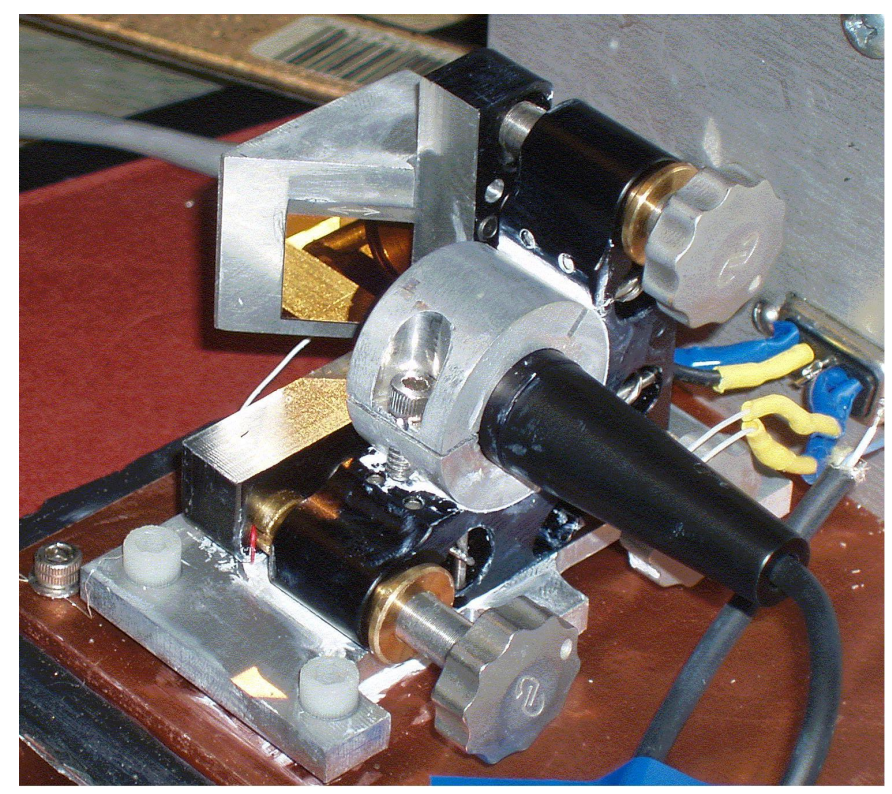

Figure 2.3: The cavity arrangement of an external cavity diode laser in Littrow configuration. The laser output is incident on a partially reflective diffraction grating. The 0th order beam is used for the experiment, while the 1st order beam is fed back into to laser cavity, thereby selecting the operating frequency.

\subsubsection{External Cavity Diode Laser (ECDL)}

The ECDL employs optical feedback from a diffraction grating to drive the output of a laser diode at a single frequency (Fig. 2.3). In the Littrow configuration, the first order diffracted beam is coupled back into the laser diode and the zeroth order reflection is used as the output beam.

The first diffraction order contains a spatially dispersed spread of frequencies from which a narrow band can be selected for feedback into the laser diode by the incident grating angle. In the design, the grating is mounted to a modified L-bracket mirror mount. The knobs on the mount enable a rough adjustment of the grating angle and are used primarily to direct the feedback signal into the diode. For fine tuning of the frequency we use a piezoelectric transducer that is inserted into the horizontal leg of the mount. Applying an external voltage to 


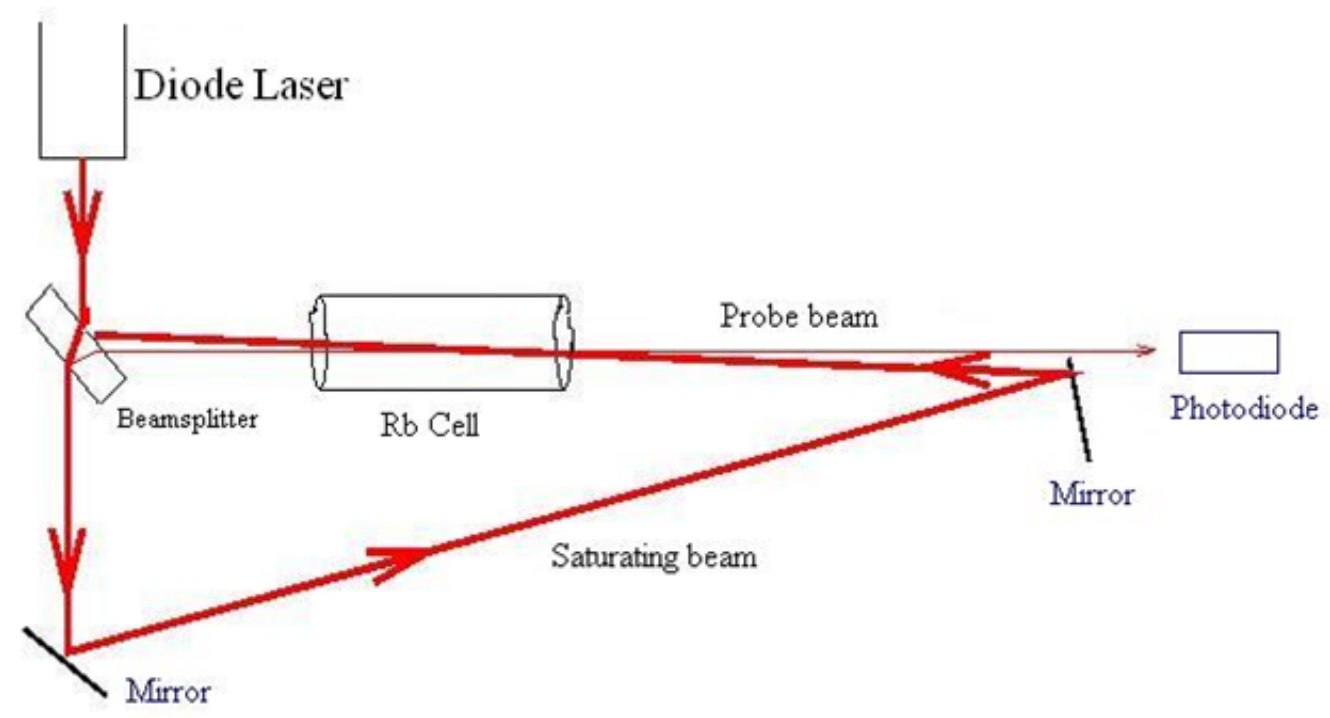

Figure 2.4: Experimental setup of a saturated absorption spectrometer. From reference [21].

the transducer produces a change in its length, thus varying the grating angle, and thereby forces the diode to operate at the frequency of the feedback signal.

\subsubsection{Saturated Absorption Spectroscopy}

The laser diode is very sensitive to changes in its temperature and current, which can cause the operational frequency to drift over time. Also, any sort of mechanical disruption (e.g. being bumped) may alter the frequency. It is therefore important to monitor and stabilize the operating frequency of the ECDL. We use saturated absorption spectroscopy to achieve this.

In our setup of a saturated absorption spectrometer the output of the laser is incident on a beam splitter which separates the beam into two beams, the probe and the pump beams (Fig. 2.4). In operation the pump beam is a much more intense beam than the probe. The probe is directed through a cell of rubidium gas and the pump beam is directed so as to counterpropagate the probe beam path. 
Consider the Doppler broadened spectrum of rubidium 85 and 87 (Fig. 2.5). The resulting broadening is due to the Doppler effect: the laser frequency in the rest frame of the atoms is different from the laser frequency in the laboratory frame. Thus atoms moving with some relative velocity blue or red shift offresonant frequencies into resonance and undergo absorption. The advantage of the saturated spectroscopy technique is that it counteracts the effect of Doppler broadening.

Consider a particular transition occurring in stationary atoms in the spatial region of both the saturating beam and probe beam. Because these atoms move with zero velocity there is zero Doppler shift and therefore they interact with both counter-propagating beams. The more intense saturating beam depletes the ground state of the transition and therefore "saturates" it. This allows the less intense probe beam to pass without engaging this particular transition. As a result the collected spectrum of the "saturated" probe beam, still characterized by a broad signal, will have on it superimposed the newly resolved hyperfine peaks corresponding to the saturated transition (Fig. 2.5). As stated, we use the

${ }^{85} \mathrm{Rb} F=3 \rightarrow F^{\prime}=4$ transition line to stabilize the frequency of our trapping laser, and the $F=2 \rightarrow F^{\prime}=3$ for the repumper.

\subsubsection{Injection Locking}

The magneto-optical trap requires a spectrally pure beam source of sufficient laser power to load the trap. The single-beam monochromatic source provided by the ECDL must be used for all three orthogonal beams and as a result the output power is reduced by a factor of three. Power loss is also attributed to the external cavity operation: that is, part of the power is lost when the diffracted beam is fed back into the diode laser. This indicates that trapping laser power 

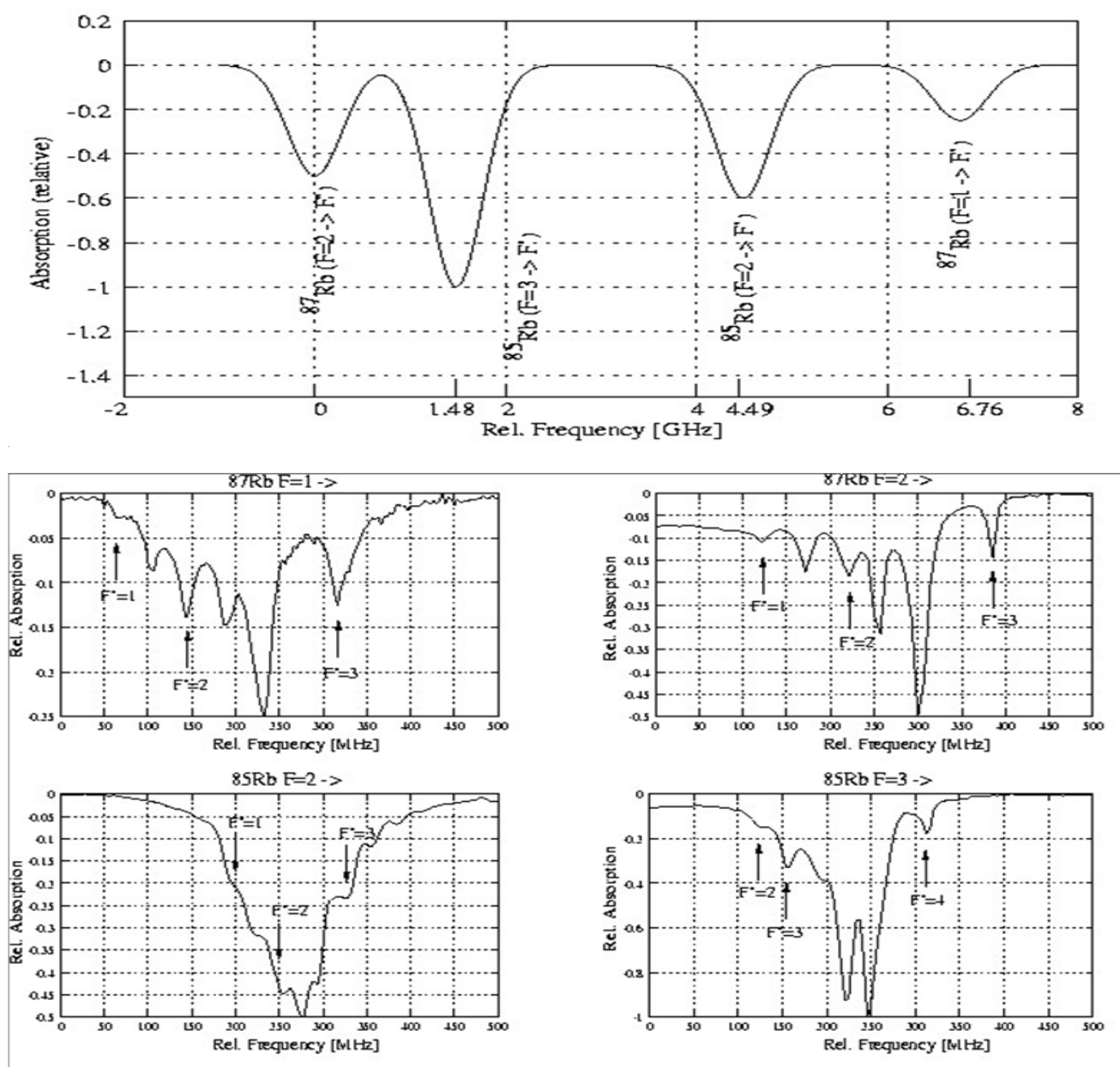

Figure 2.5: The relative absorption of ${ }^{85} \mathrm{Rb}$ and ${ }^{87} \mathrm{Rb}$. The top-half of the trace displays the relative absorption as a function of frequency of the free running beam by atoms in a $\mathrm{Rb}$ gas. The lower half displays the resolved Doppler-free hyperfine spectra. From reference [21].

is severely limited by single-laser operation. (Also, the drawbacks associated to spectral width and frequency instability of ECDL's impose limits). One could circumvent this issue by using a high-power laser, but would then be more susceptible to mechanical and thermal influences. In principle, one could also construct a lower power source and amplify it using a high power amplifier. But this is also problematic in that it leads to unavoidable sources of noise (e.g. amplifier noise and intensity noise). 
We use an injection-locking amplifier system to counteract the spectral limitations described above. In this system the high power is produced by injecting the output of the ECDL into the cavity of a free running diode laser. We call the ECDL the master laser and this second laser the amplifier laser. Provided that the frequencies of the master laser and the amplifier laser are sufficiently close, the injection forces the amplifier laser to operate at the injected frequency. This is a consequence of the fact that diode lasers are very sensitive to optical feedback. Note that the higher the injected power, the larger the allowable offset of frequencies is between the two lasers. Also, the respective beams must be parallel and overlap in order to optimize the optical feedback. The frequency of the amplifier laser is monitored by a spectrum analyzer. Both the trapping laser and repumper laser use the injection-locking amplifier system.

\subsection{Experiments on Rydberg Atoms}

The previous section describes how we capture ${ }^{85} \mathrm{Rb}$ atoms in a magneto-optical trap. In our experiments, we excite these atoms to high principal quantum number Rydberg states using a dye-amplified pulse laser, and observe the spontaneous evolution of Rydberg atoms to plasma. Alternatively, we directly produce a plasma by photoionizing the trapped sample of ${ }^{85} \mathrm{Rb}$ atoms with a Littmantype dye laser. For both of these procedures, the $5 \mathrm{p}_{3 / 2}$ state atom is the effective ground state of the system. We do Rydberg atom spectroscopy by probing Rydberg-Rydberg transitions using a millimeter-wave spectrometer. The mmwave spectra help us to diagnose the Rydberg state density produced by dyeamplified pulse laser excitation. 


\subsubsection{Pulsed Dye Lasers}

Many cold Rydberg atom experiments use pulsed laser systems to produce the Rydberg atom population. But when exciting Rydberg states using $\approx 10$ ns dye lasers, shot-to-shot changes in the Rydberg population occur as a result of the poor overlap between the longitudinal modes of the dye laser and the transition spectral width. That is, generally, in each laser shot, several laser modes will oscillate within a bandwidth of $\approx 0.1 \mathrm{~cm}^{-1}(3 \mathrm{GHz})$, the width of each mode being $\approx 100 \mathrm{MHz}$. Now from one shot to the next, the intensities of each mode will change, and more significantly, the frequency of each mode will shift. Therefore, not only is the spectral overlap of a laser mode with the transition poor, but it changes on a shot-to-shot basis, causing significant fluctuations in the Rydberg atom population. Now, the Doppler width of transitions in $100 \mu \mathrm{K} \mathrm{Rb}$ atoms is less than $1 \mathrm{MHz}$, so therefore the spectral width of most transitions is limited by state lifetimes of the upper and lower states. For the $5 \mathrm{p}_{3 / 2}$ state (the upper level of the trapping transition) to a Rydberg state transition, the limit is the $5 \mathrm{MHz}$ natural width of the $5 \mathrm{p}_{3 / 2}$ state. Therefore the key to removing the shot-to-shot instability is to produce a laser source that is spectrally stable, centered on the transition frequency, and broad enough to maintain sufficient overlap with the transition linewidth despite any spectral drifts that may occur. Note that some experiments try to counter these drawbacks by collecting data using a binning approach. Specifically Li et. al. [1] set gated integrators over the plasma electron (or ion) signal and field ionization signal and record both for each shot of the laser. This signal is assumed to represent the initial Rydberg state population. Thus by monitoring the Rydberg population in this way, Li et. al. determined how the initial Rydberg state populations effect plasma formation. Their results are described in section 1.2. 


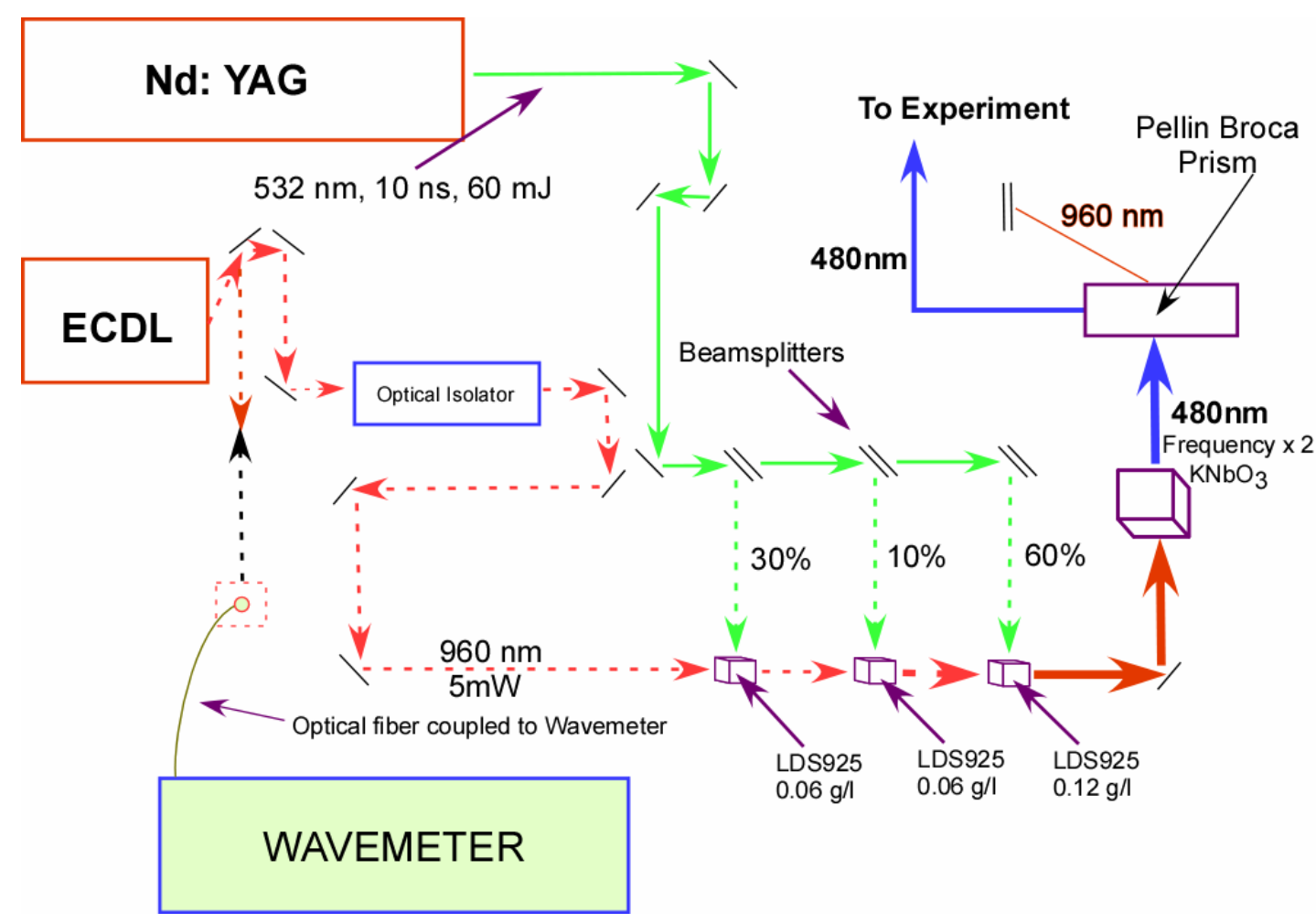

Figure 2.6: The pulsed-amplified CW laser system. The $960 \mathrm{~nm}, 5 \mathrm{~mW}$ output of the ECDL is directed through an optical isolator and incident on three LDS925 diode cells where it is amplified, pumped by the second harmonic $532 \mathrm{~nm}$ source from a Nd: YAG 10ns pulse laser. The amplified beam is frequency doubled in a $\mathrm{KNbO}_{3}$ crystal resulting in the $480 \mathrm{~nm}$ light source at $20-40 \mu \mathrm{J}$ per pulse.

Because we are interested in the Rydberg to plasma dynamics (and subsequent features after plasma formation), it is important to maintain a high Rydberg signal. We have circumvented the instability by constructing a narrow linewidth, frequency doubled, pulsed-amplified CW laser system (Fig. 2.6). And we are able to obtain relatively stable shot-to-shot Rydberg state populations as a result.

Our system is based on a home-made ECDL operating around $960 \mathrm{~nm}$. The output power from the laser cavity is $5 \mathrm{~mW}$, and the spectral width is $\approx 10 \mathrm{MHz}$. Operation of the external cavity laser is monitored with a scanning confocal etalon, and the wavelength is measured using a commercial wavemeter. After passing through a $40 \mathrm{~dB}$ optical isolator, the light from the diode laser is amplified 
in three dye cells (LDS 925) pumped with the $532 \mathrm{~nm}$ light from a Nd:YAG laser and then frequency doubled to $480 \mathrm{~nm}$ in a $\mathrm{KNbO}_{3}$ crystal. The doubled output is $20-40 \mu \mathrm{J}$ per pulse, with a spectral width that is Fourier transform limited by the $10 \mathrm{~ns}$ YAG pulse to approximately $100 \mathrm{MHz}$. Using this system, the shot-to-shot variation in the Rydberg signal is less than $\pm 10 \%$. The laser can be tuned to a transition using the wavemeter, and fine tuning is done by changing the voltage to a piezoelectric crystal that shifts the grating in the ECDL.

We use a second pulsed laser to directly photoionize the cold atoms (Fig. 2.11). This laser is a Littman-type dye laser, and is pumped using the third harmonic $355 \mathrm{~nm}$ light from the Nd:YAG laser. It excites atoms in the $5 \mathrm{p}_{3 / 2}$ state directly to the continuum, and therefore frequency fluctuations cause no shot-toshot variation in the number of ions formed, although there may be some small fluctuation in the ion density due to the energy per pulse. In our experiments we investigate the effect of embedding Rydberg atoms in a pre-existing plasma on the plasma electron energy spectrum. We first fire the Littman laser to create a plasma, and then, approximately 15 ns later, the frequency doubled, pulseamplified cw laser system is used to excite to a specific Rydberg state. Using the narrow bandwidth laser system, we can create a maximum Rydberg density $\approx 1 \times 10^{10} \mathrm{~cm}^{-3}$. However, our estimates of absolute Rydberg atom population densities have an uncertainty of a factor of 3 , though relative populations can be measured to $10 \%$.

\subsubsection{Millimeter-wave Spectroscopy}

The large separation between the electron and the ionic core in high- $n$ Rydberg atoms results in large electric dipole moments. At high densities, these strong dipole moments are responsible for strong van der Waals coupling between neigh- 
boring Rydberg atoms [16]. Excitation to high- $n$ Rydberg states in a dense sample of atoms allows us to turn on these strong dipole-dipole interactions.

In recent investigations involving dense samples of atoms by Lukin et. al. it is suggested that Rydberg atoms will block further resonant excitation due to the perturbations of energy levels as a result of dipole-dipole interactions, a process called "dipole blockade" [17]. And this field has gathered much interest in quantum information processing. According to Jaksch et. al., the large interaction energy required to perform fast gate operations can be provided by the dipole-dipole interaction of atoms excited to low-lying Rydberg states [19].

Our work focuses on the spectral line broadening of transitions between high$n$ Rydberg states that occur as a result of the van der Waals coupling. This result was first confirmed by Raimon et. al [16] and subsequently by Afrosheh et. al. [18]. We probe the dipole-dipole interactions using mm-wave transitions between Rydberg states, and in so doing, make an adequate diagnosis of our Rydberg densities: if we can achieve the spectral broadening proposed by Raimon et. al [16] and Afrosheh et. al. [18] then we have achieved the density regime necessary to explore Rydberg to plasma formation and successive plasma dynamics.

Consider a system of two neighboring high- $n l$ atoms, $|n l, n l\rangle$, coupled by their respective magnetic dipole moments, $\mu_{1}$ and $\mu_{2}$ (Fig. 2.7). Raimon et. al. suggest that the perturbation of one atom can be calculated as if it were interacting only with its closest neighbor (which can be considered to be at rest for the purposes of this discussion) [16]. Using the appropriate microwave photon energies, $|n l, n l\rangle$ states can be coupled to neighboring $\left|n^{\prime} l \pm 1, n^{\prime \prime} l \pm 1\right\rangle$ states. As described by Fig. 2.8, at long distances the $|n l, n l\rangle$ to $\left|n^{\prime} l \pm 1, n^{\prime \prime} l \pm 1\right\rangle$ transition produce spectra of an isolated atom, one that is unaffected by the dipole-dipole forces. When the interatomic space is reduced, a strong coupling exists, via 


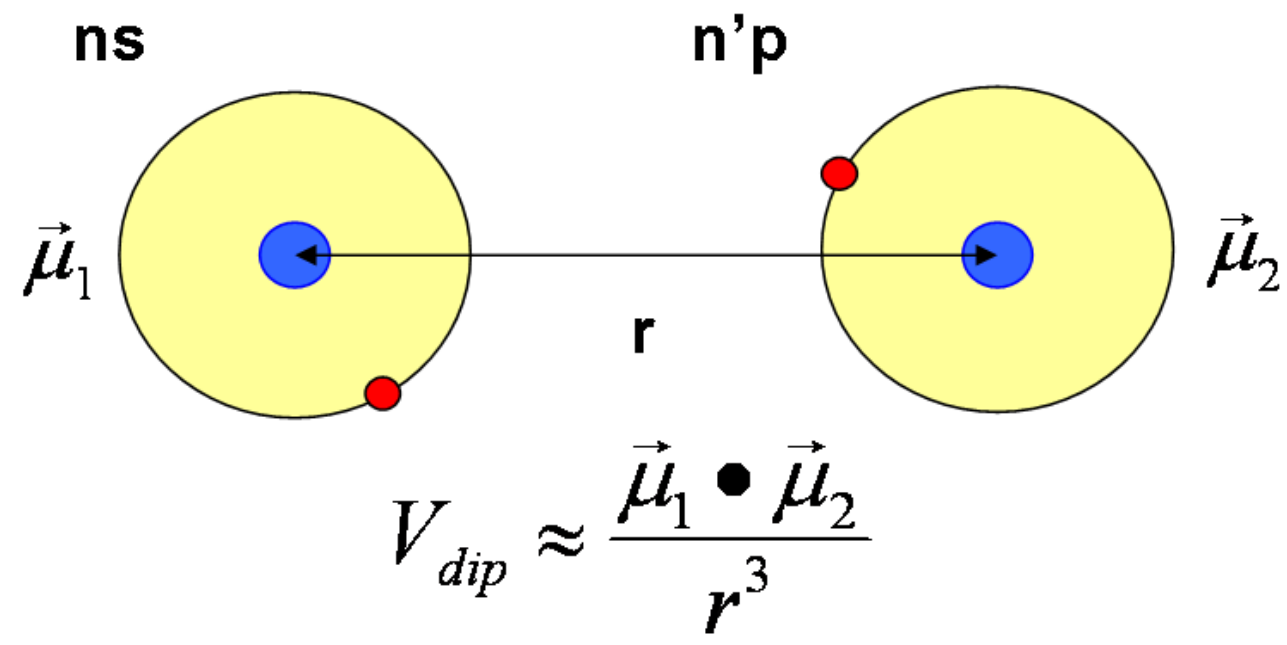

Figure 2.7: Dipole interaction and associated potential energy $V_{d i p}$ between pairs of Rydberg atoms due to their respective electric dipole moments $\mu_{1}, \mu_{2}$, and interatomic separation $r$.

the dipole-dipole interaction, between the $|n l, n l\rangle$ states and the closely lying $\left|n^{\prime} l \pm 1, n^{\prime \prime} l \pm 1\right\rangle$ states. As a result, the states experience strong van der Waals forces that produce a manifold of energy levels, two of which are shown in Fig. 2.8 [16]. When we probe the transitions between these energy levels with the appropriate microwave photon energies the resultant spectrum is broadened by the different transition frequencies in the manifold. Note that, in the situation where one of the atoms is in a low- $n$ state, the interaction is negligible due to the smaller dipole moment, and the energy levels to a good approximation are $r$ independent [16].

Millimeter wave spectroscopy is a powerful tool that is used to reveal the energy level structure of Rydberg atoms, to induce population transfer between different Rydberg states, and to selectively detect small populations of atoms in specific Rydberg states. We use a mm-wave source that can produce approximately $10 \mathrm{~mW}$ of radiation in ranges of $20-40 \mathrm{GHz}$, and $53-110 \mathrm{GHz}$. The source 


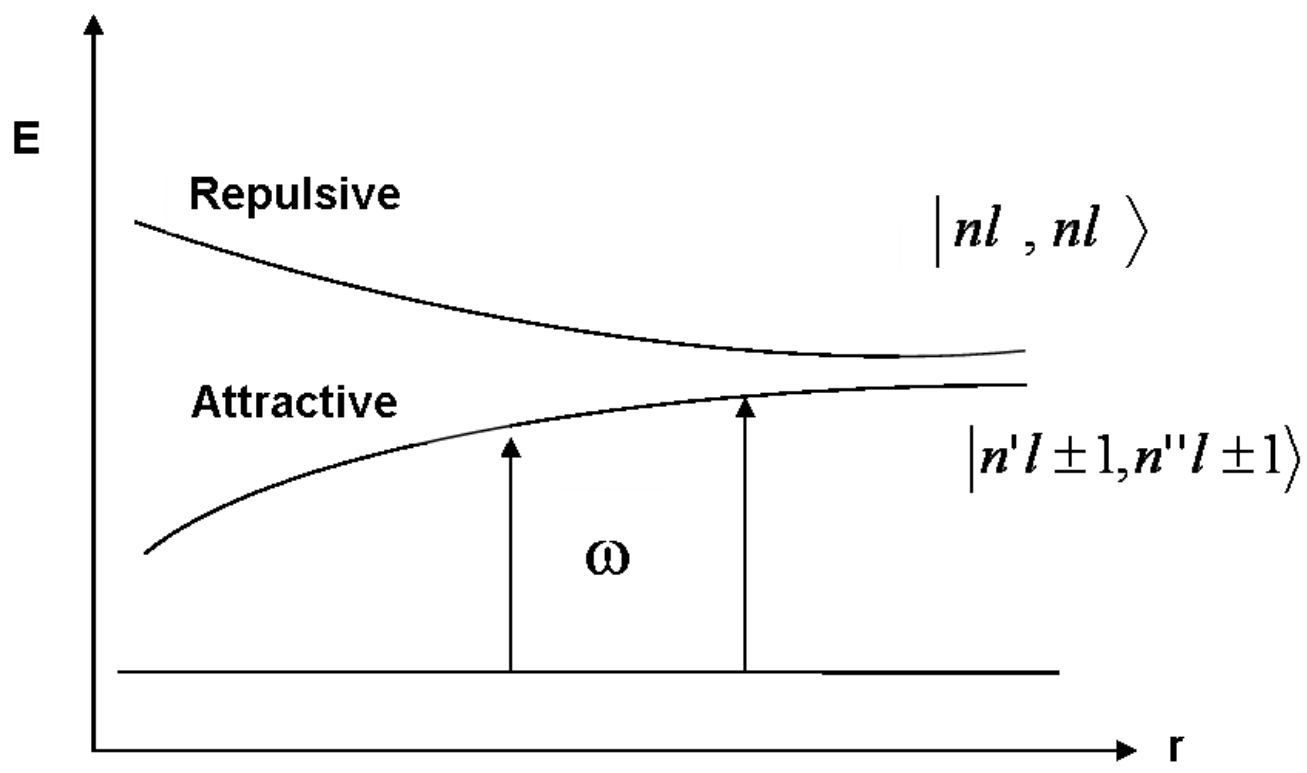

Figure 2.8: Schematic diagram of the perturbation of energy levels in neighboring ${ }^{85} \mathrm{Rb}$ atoms in high- $n$ Rydberg states a distance $r$ from each other. We drive transitions using mm-wave fields at frequencies $\omega$ to probe the repulsive and attractive van der Waals forces. At distances where the interaction energy is strong the spectral lines corresponding to transitions between neighboring $\mathrm{nl}$ and $n(l \pm 1)$ Rydberg states broaden.

is based on a $10 \mathrm{MHz}-26.5 \mathrm{GHz}$ sweep oscillator capable of generating $12 \mathrm{dBm}$ from 10-20 GHz, $10 \mathrm{dBm}$ from 20-26.5 GHz (Hewlett-Packard HP83630). The output of this source is gated to make pulses of arbitrary length (typically 1-10 $\mu \mathrm{s}$ ), and then amplified and/or frequency multiplied using active or passive doublers and triplers. Using the appropriate combination of waveguides, isolators, and attenuators (those appropriate for the frequencies in use) the mm-waves are directed into the MOT via horn and an off-axis paraboloid reflector. This source is used with the dye-amplified diode laser system to obtain the spectrum of the $39 \mathrm{~s}_{1 / 2} \rightarrow 39 \mathrm{p}_{3 / 2}$ transition.

Cold atoms are excited to the $39 \mathrm{~s}_{1 / 2}$ state by the dye-amplified diode laser. At a delay of $1 \mu \mathrm{s}$, a $2 \mu \mathrm{s}$-long pulse of mm-waves excite some of the atoms to the $39 \mathrm{p}_{3 / 2}$ state. After $1 \mu$ s we selectively field ionized the $39 \mathrm{p}_{3 / 2}$ state but 
(a)

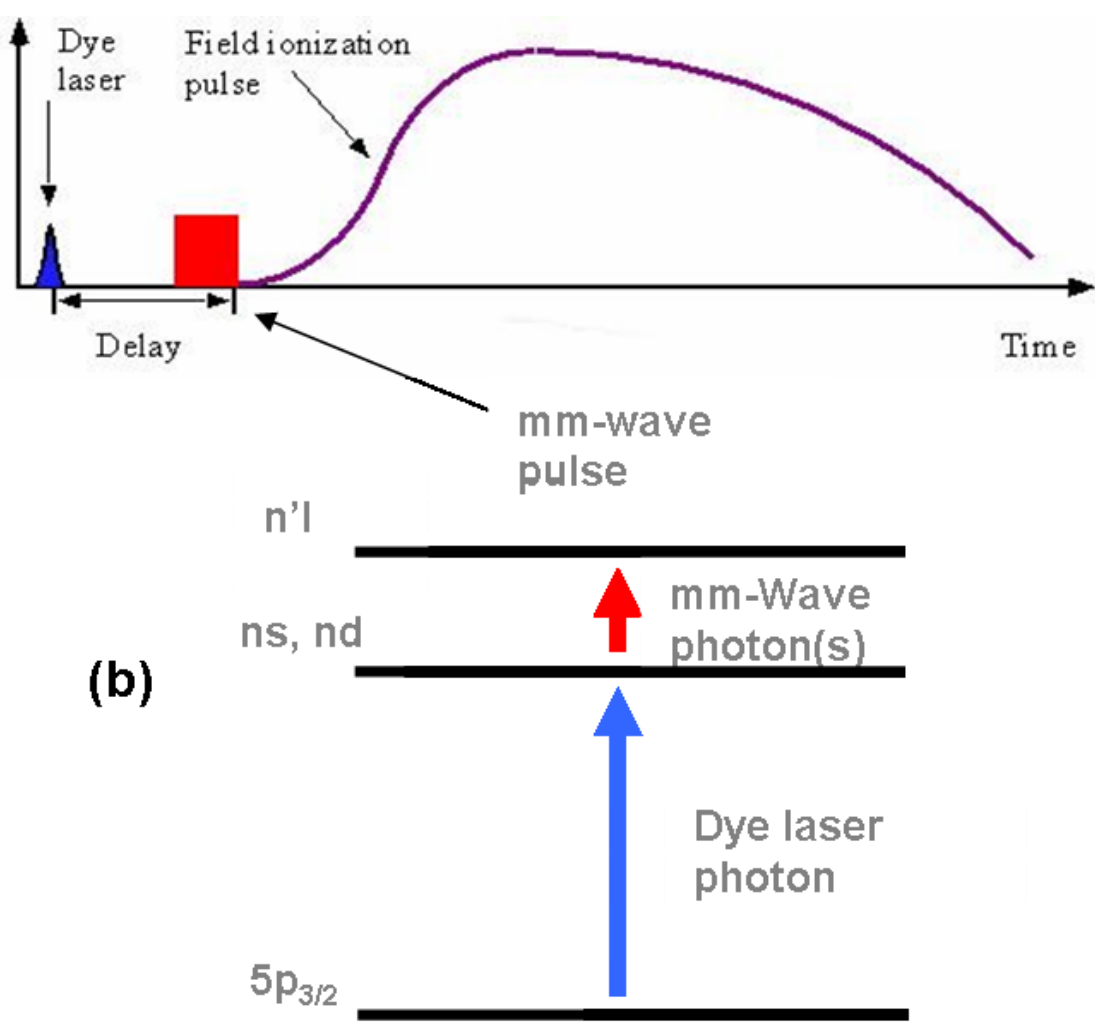

Figure 2.9: (a) Diagram of the time sequence for the mm-wave spectroscopy of Rydberg atoms. (b) Energy level diagram. The $n s$ or $n d$ state is populated by a $10 \mathrm{~ns}, 480 \mathrm{~nm}$ laser pulse. Millimeter waves excite a $n^{\prime} l$ state by either a single photon transition, or a two photon transition.

not the $39 \mathrm{~s}_{1 / 2}$ state. The time-resolved signal of electrons detected on the microchannel plate (MCP) detector is acquired via gated integration. Under the control of LabView the mm-wave frequency can be scanned while the signal is being acquired [26].

In this experiment we vary the Rydberg density by varying the number of atoms initially stored in the trap. This is done by combining a half-wave plate with a polarization cube to change the intensity of the trapping laser. With a less dense population of atoms stored in the trap we therefore excite fewer atoms to the Rydberg state, thereby reducing of Rydberg density. The results of the experiment are shown in Fig. 2.10. We see that at our highest density of 

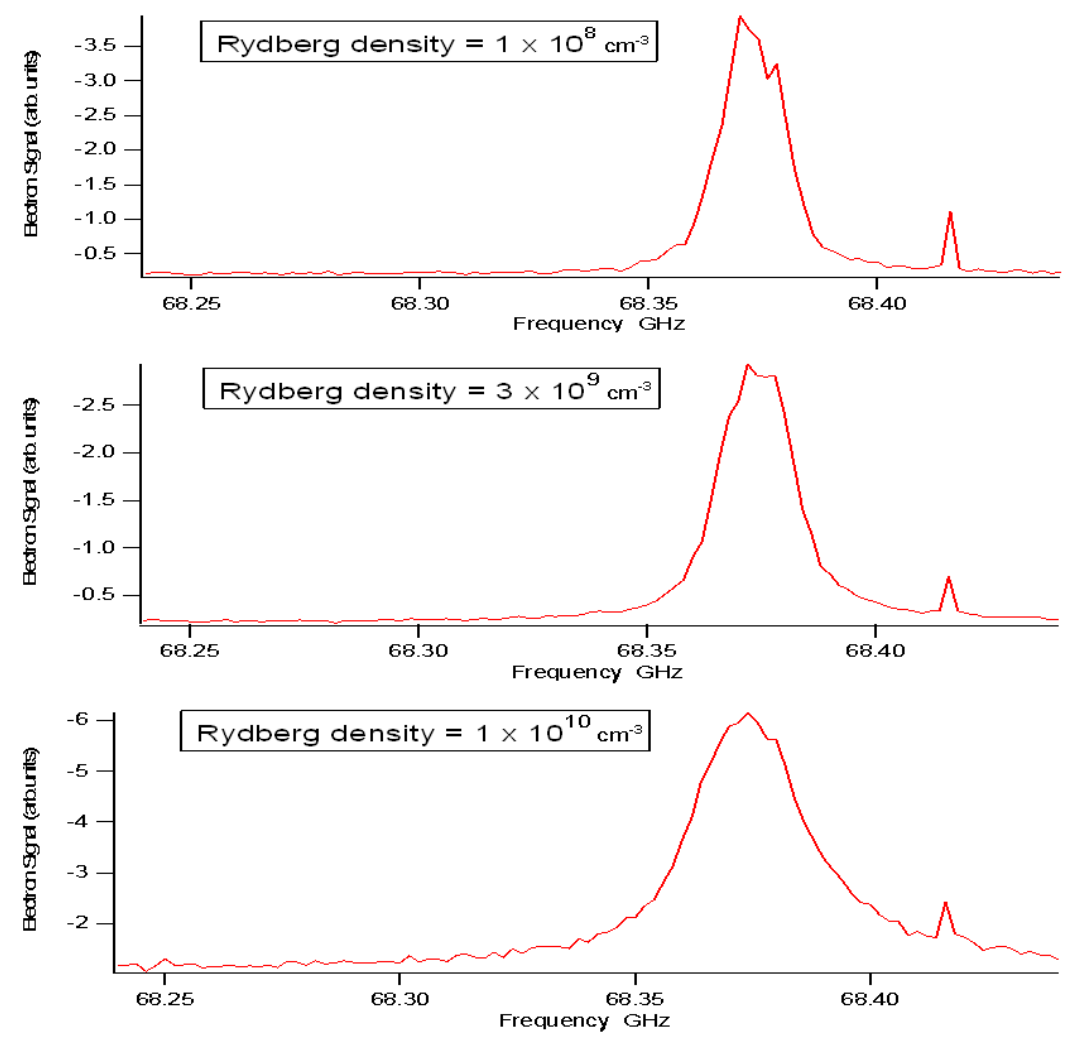

Figure 2.10: Millimeter wave spectrum at different Rydberg densities. The signal is from the field ionization of the $39 \mathrm{p}_{3 / 2}$ state. Shown are the spectra at Rydberg densities $1 \times 10^{8} \mathrm{~cm}^{-3}, 3 \times 10^{9} \mathrm{~cm}^{-3}$, and $1 \times 10^{10} \mathrm{~cm}^{-3}$. As densities are increased the spectrum broadens, signifying the broadening effect expected due to the dipole-dipole interaction.

$1 \times 10^{10} \mathrm{~cm}^{-3}$ the signal is in fact much broader than that of the lower densities presented. And this broadening is characteristic of the densities needed to observe the Rydberg to plasma evolution and the subsequent dynamics that result.

\subsubsection{State Selective Field Ionization (SSFI)}

One of the most explored properties of Rydberg atoms are their sensitivity to external electric fields and magnetic fields. Often experiments have to find means of removing weak stray fields to prevent the perturbations that result from the strong interaction Rydberg atoms have with those fields (i.e. magnetic shield- 


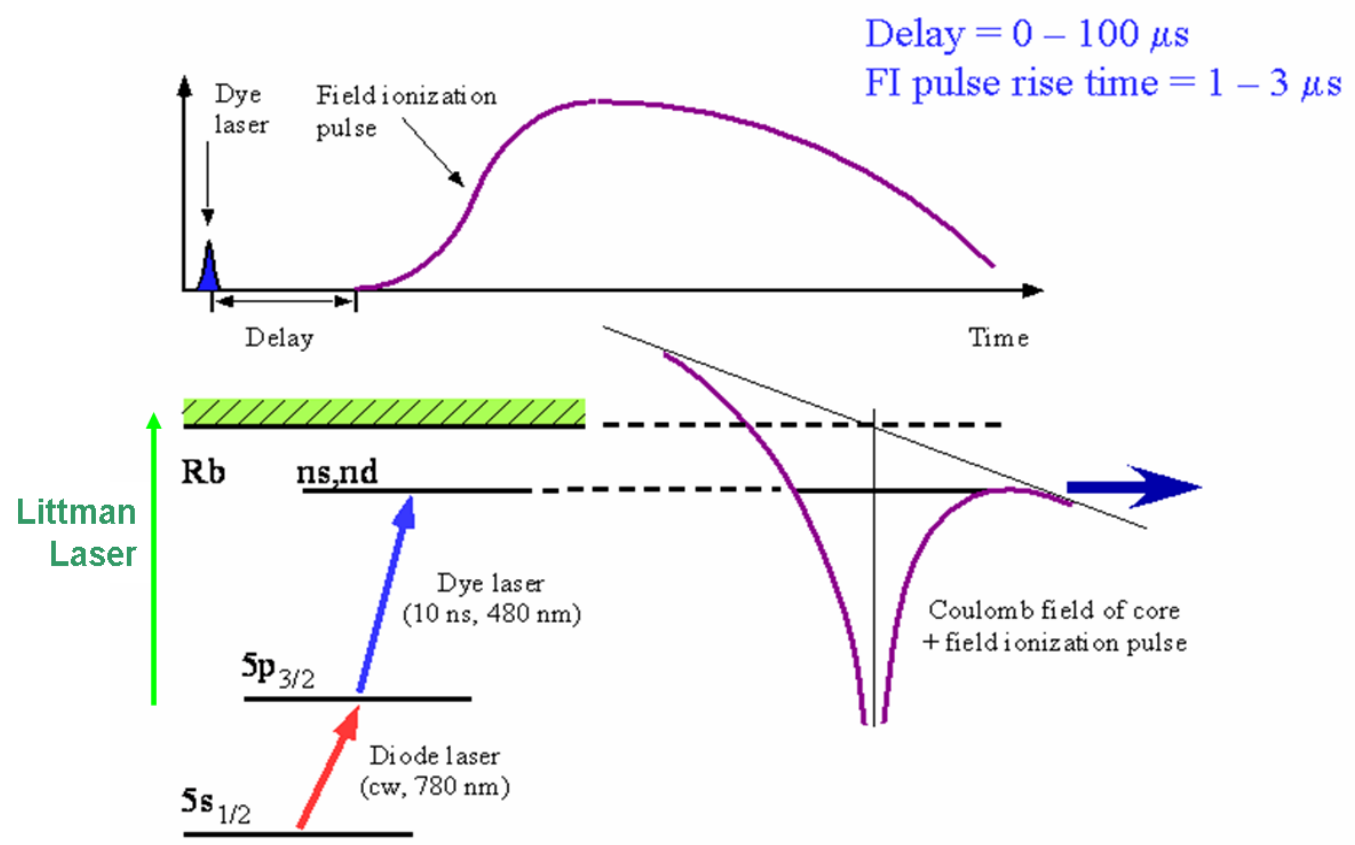

$\mathrm{Rb}(\mathrm{Cs}): 300 \mu \mathrm{K}(140 \mu \mathrm{K}) ; 1.5 \times 10^{7}$ $\left(5.0 \times 10^{7}\right)$ atoms; maximum Rydberg density $1 \times 10^{10} \mathrm{~cm}^{-3}$

Figure 2.11: The general sequence on events during our experiments. Rubidium atoms are trapped and stored in the $5 \mathrm{p}_{3 / 2}$ state. Using the narrow bandwidth laser system we can excite $\mathrm{Rb} 5 \mathrm{p}_{3 / 2}$ atoms into high $n s$, nd Rydberg states. We have achieved a maximum Rydberg density of $1 \times 10^{10} \mathrm{~cm}^{-3}$. The Littman-type dye laser, pumped by the third harmonic $355 \mathrm{~nm}$ light of the Nd:YAG is used to directly excite $5 \mathrm{p}_{3 / 2}$ Rb atoms above the ionization threshold, thereby creating a plasma. We use state-selective field ionization to detect Rydberg states and the electrons (or ions) that result from the evolution of the system. The figures for Cs are noted as a reference to experiments that use Cs as the primary element.

ing). State-selective field ionization (SSFI) takes advantage of the electric field interaction, namely the Stark effect, as relatively modest fields not only perturb the Rydberg levels, they even lead to ionization of Rydberg atoms. Coupled with a micro-channel plate (MCP) electron multiplier detector, SSFI provides an experimentally feasible means for detection of Rydberg states.

Fig. 2.11 shows the potential that a Rydberg atom experiences in the presence of an electric field. This potential is classically modeled by Gallagher [5] as 
follows. Consider a $\mathrm{H}$ atom (or a hydrogen-like Rydberg atom) with its nucleus at the origin in an electric field directed along the $z$-axis. The potential experienced by the electron is a sum of the Coulomb term and the term due to the electric field given by $V=-\frac{k}{|z|}+E z$, as shown in Fig. 2.11. Electrons with energies lower than the local maximum are bound. Equating the energy of the Rydberg state to this local maximum leads to a classical field for ionization of the Rydberg state with principal quantum number $n$, given by $E=1 / 16 n^{4}[5]$.

In our experiment we excite Rydberg atoms and at a specified delay (Fig. 2.11) we ionize them using a $\approx 1000 \mathrm{~V}, 1 \mu$ s rise time voltage pulse applied to the transparent meshes inside the vacuum chamber. The resulting electrons or ions are swept to the MCP. If a plasma is present the ions or electrons from the plasma are also driven to the MCP. The arrival time to the detector is determined by the time at which the voltage pulse reaches the electric field needed to ionize a particular Rydberg state. Therefore, in general, electrons arriving at later times are coming from more deeply bound Rydberg states, though the detection is also sensitive to different angular momentum states. The time-of-flight spectra therefore reveal information on the population distribution of Rydberg states in the sample and give information about the energy distribution of the plasma electrons.

\subsubsection{Timing Sequence}

The timing sequence of the experiment, also represented in Fig. 2.11, is controlled by two DG535 Digital Delay and Pulse Generators. One acts as the master clock, providing a trigger at $20 \mathrm{~Hz}$. This triggers the YAG laser that pumps the narrow line width system and Littman dye laser. The second DG535 generates pulses for the millimeter wave system, the oscilloscope and boxcar integrators, and the 
field ionization pulse. In operation the millimeter wave function is optional and is removed unless doing millimeter wave spectroscopy. It is used primarily in the mm-wave experiments detailed above in section 2.3.2, and in our Rydberg lifetime measurements. 


\section{Chapter 3}

\section{Experiments and Results}

This section describes our preliminary investigation of the Rydberg to plasma evolution and the possibility of using Rydberg atoms to influence the plasma temperature. The results of the second investigation show possible evidence that Rydberg atoms do, in fact, stabilize the plasma.

\subsection{Evolution of Rydberg Atoms to Plasma}

This part of the experiment uses the narrow line width frequency doubled dye amplified diode laser system to excite a selected Rydberg state. Tests are carried out to test the system's ability to produce dense samples of Rydberg atoms. The results of this investigation also issues information on the behavior that characterizes the Rydberg to plasma evolution. The results are shown in Fig. 3.1. These results are previously reported work in Ref. [1]. As can be seen a robust plasma forms as early as $1 \mu$ s after the Rydberg state is populated. This plasma is quite deeply bound, as it takes an electric field of $78 \mathrm{~V} / \mathrm{cm}$ to remove the electron from the plasma (a field strength sufficient to field ionize a state of 


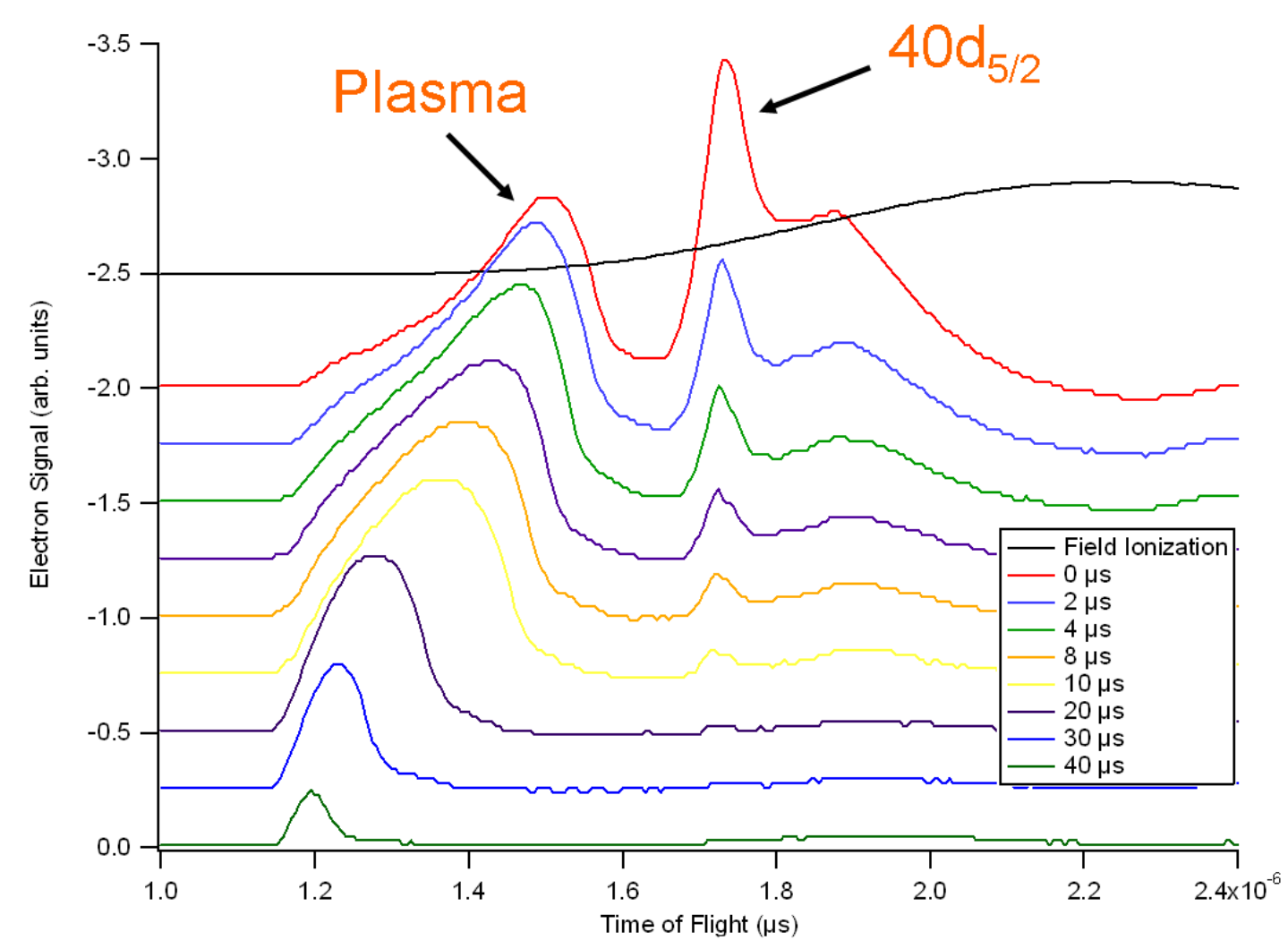

Figure 3.1: Electron time-of-flight signal from the field ionization (FI) of a dense sample of $40 \mathrm{~d}_{5 / 2} \mathrm{Rb}$ atoms, and the plasma to which the state evolves. The Rydberg atom density is $\approx 1 \times 10^{10} \mathrm{~cm}^{-3}$. The signal shown is the plasma evolution as a function of delay between the excitation laser and application of the FI pulse. The top-most signal is the field ionization pulse. The vertical scale is the same for all graphs except the FI signal.

quantum number $n=45)$.

It is important to note that the FI signal is not capable of distinguishing highly excited states in the continuum from a plasma signal (they are essentially equivalent). Also, it is difficult to extract quantitative information from the plasma using this technique because as we remove electrons the ones left behind become more tightly bound, thereby changing the plasma dynamics and subsequent measurement of an electron temperature. Roberts et. al. provide a technique that circumvents this affect, probing the electron energy distribution 
without significantly affecting the plasma dynamics [25]. After a desired delay an electric field is applied to the sample. This field will lower the lip of the potential well confining the electrons, and energetic electrons are freed. In order to avoid dynamic changes in the confinement, the fraction of atoms spilled is kept small enough so that the ratio of electrons to ions is not altered substantially. The spilling curves are reported in [25]. Roberts et. al. construct a model that allows extraction of the electron temperature from the spilling curves, and we intend to follow this model in our measurements of the electron temperature.

At long delay times, the plasma expands due to the Coulomb repulsion of the ions and the Rydberg signal decreases significantly. As the plasma expands the more energetic electrons are able to escape because the trap strength decreases as the potential well becomes shallower. The plasma signal becomes smaller and moves to lower flight times, corresponding to smaller plasma quenching electric field strengths. Another important feature is the hump that occurs to the right of the $40 \mathrm{~d}_{5 / 2}$ signal. This represents ionization of more deeply bound $n l$-states. Population in these states is necessary to maintain conservation of energy, the energy balance that enables the ionization of Rydberg atoms and the formation of the plasma. We finally observe that the plasma is less bound when the $40 \mathrm{~d}_{5 / 2}$ Rydberg state effectively vanishes. This suggests that one can use Rydberg atoms to stabilize a pre-existing plasma.

We have also observed the density dependence of the evolution process, the results of which are shown in Fig. 3.2. As can be seen, the Rydberg sample only evolves to plasma at the highest densities. At low densities we do observe a redistribution into more deeply bound $n l$-states, and this may lead to ionization, but it is likely that the excess positive charge density of the ions is never sufficient enough to form a plasma. 

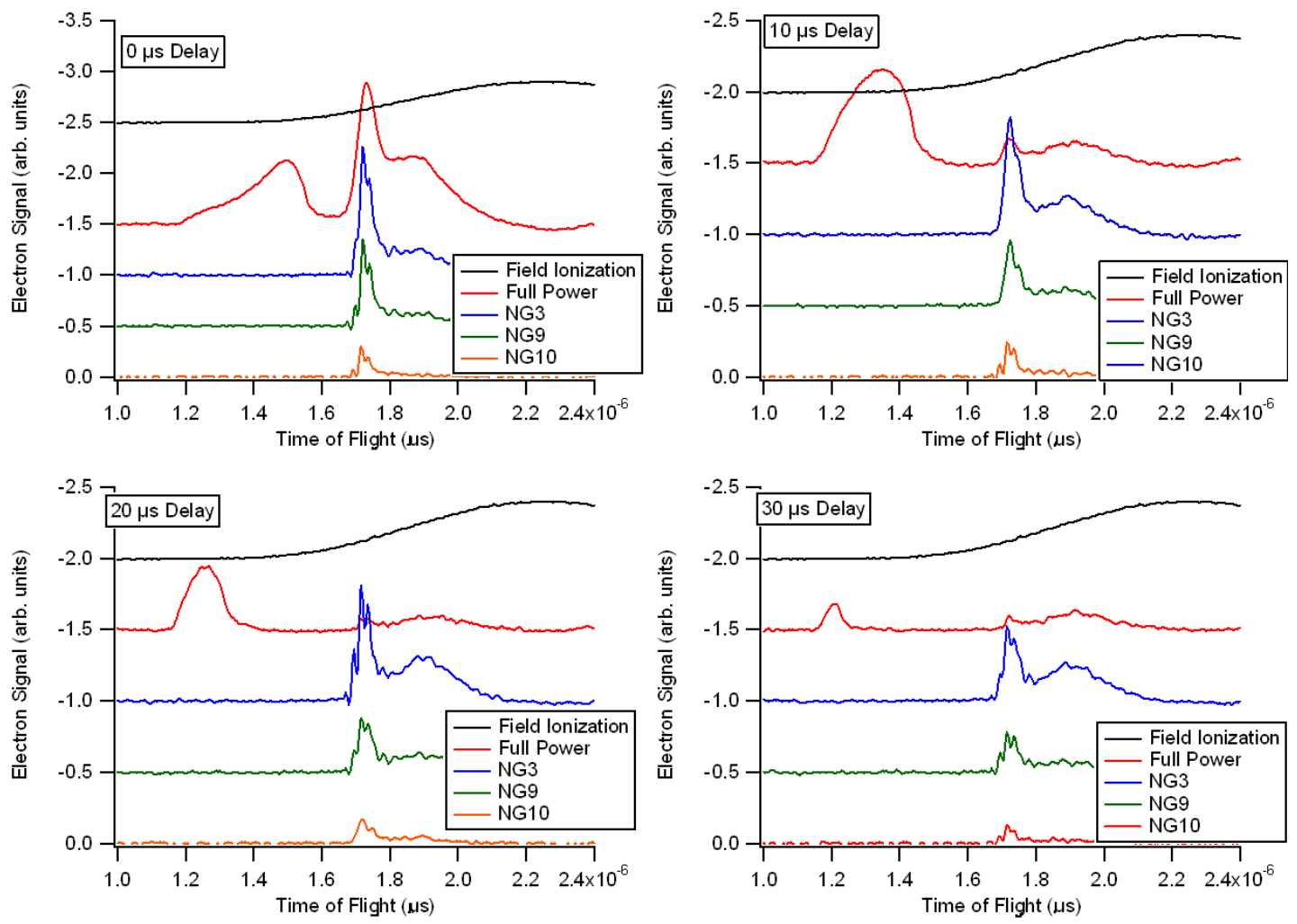

Figure 3.2: Electron time of flight data showing the dependence the plasma evolution has on the Rydberg atom density. We use neutral density (ND) filters to vary the Rydberg atom density. Full laser power, ND3, ND9, and ND10, correspond to densities $1 \times 10^{10} \mathrm{~cm}^{-3}, 2 \times 10^{9} \mathrm{~cm}^{-3}, 1 \times 10^{9} \mathrm{~cm}^{-3}$, and $3 \times 10^{8}$ $\mathrm{cm}^{-3}$ respectively. The topmost signal is the FI signal, and all subsequent curves (top-down) represent the plasma evolution at decreasing Rydberg densities. The vertical scale is the same for all graphs except for the FI signal.

\subsection{Two-Laser Experiment}

It has been noted above that the plasma is more stable when there is a significant Rydberg density present. It is this notion that motivates our two-color experiments. This idea stems entirely from the concerns of energy conservation during the evolution process. Electron collisions with very highly excited Rydberg states gradually decrease in binding energy and Rydberg atoms ionize. Alternatively, electron collisions with low-lying states increase their binding energies, and there- 
fore results in electron heating. In the first scenario the Coulomb space charge is unaffected and electron are cooled. In the heating regime electrons are ejected from the plasma, aiding the plasma expansion. To determine what regime we operate in, a qualitative means of determining the electron temperature is necessary. We intend to use the spilling technique demonstrated by Roberts et. al [25] for this measurement.
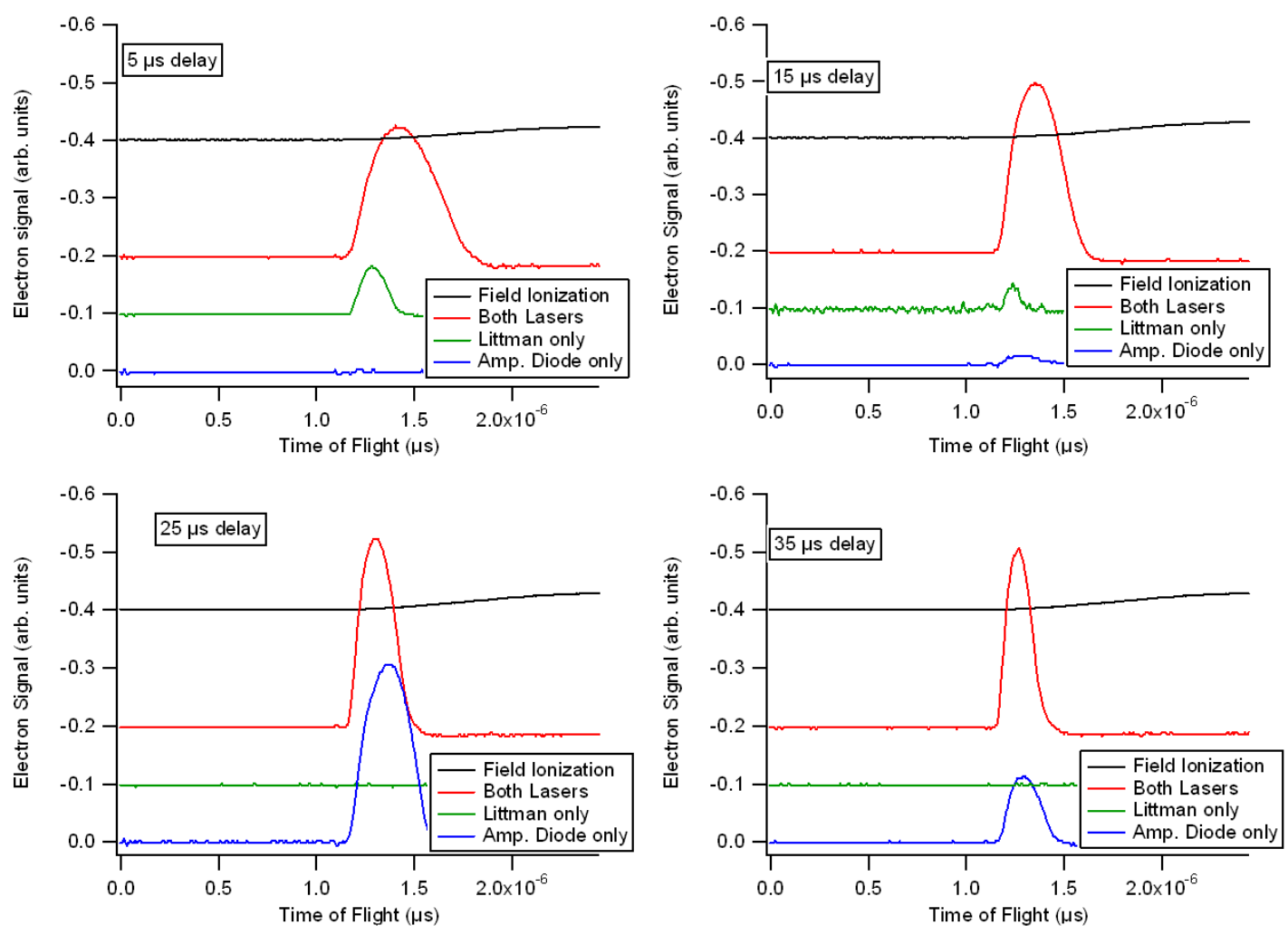

Figure 3.3: Electron time-of-flight signals from an ultra cold plasma collected in the two-color experiments. The signals shown are for different delays between the laser and FI pulse, and with- and without the presence of $40 \mathrm{~d}_{5 / 2}$ Rydberg atoms. Read top-down the signals represent the (1) FI pulse, (2) the plasma formed when using both the Littman dye laser and the narrow line width laser, (3) the Littman dye laser (no narrow line width laser), and (4) the narrow line width laser (no Littman dye laser). The vertical scale is the same for all signals except for the FI pulse and the FI pulse was not sufficiently large enough to ionize the $40 \mathrm{~d}_{5 / 2}$ state.

In these experiments, we use the pulsed Littman dye laser to photoionize the 
sample of cold $\mathrm{Rb} 5 \mathrm{p}_{3 / 2}$ atoms to create a plasma directly. At a delay of 15 ns, we used our narrow line width laser to populate the $40 \mathrm{~d}_{5 / 2}$ state from cold atoms remaining in the $5 \mathrm{p}_{3 / 2}$ state. We then observed the evolution process of the sample as a function of delay from the Rydberg excitation. These data are shown in Fig. 3.3. In these experiments, we reduced the FI pulse amplitude so that it was large enough to quench the plasma, but not large enough to field ionize the $40 \mathrm{~d}_{5 / 2}$ state. We did this to maintain as high a trap density as possible over the course of the experiment. The experiment is performed at $20 \mathrm{~Hz}$, and a significant fraction of the cold atoms are either ionized, or de-excited to a Rydberg state that was not ionized by the electric field pulse. If we field ionize the Rydberg atoms, these cold atoms are removed from the trap, which doesn't refill completely before the next laser shot. Typically, in the experiments described above, our cold atom density decreases by $30 \%$ when we field ionize the Rydberg atoms. The Rydberg atom and plasma densities we can create are therefore significantly higher when we reduce our FI pulse so that only the plasma is quenched.

The curves in Fig. 3.3 represent three independent scans (not including the field ionization signal). The uppermost curve represents the signal produced when both the Littman laser and the diode laser are in use. In this mode, we create a plasma using the Littman laser and then subsequently add Rydberg atoms with the diode laser. The other two curves respectively are the plasma signals when the only laser on is either the Littman laser or the diode laser. At $5 \mu$ s we see that, the signal for the diode laser only is flat, signifying that the Rydberg atoms have yet to evolve to plasma. At the same delay the plasma produced by the Littman laser is present. But as we increase the delay times this plasma starts to expand and vanish. Note also that as we increase the delay time the Rydberg atoms created by the diode laser evolve to plasma. The evolution of this plasma 
is described by the results in Fig. 3.1.

Looking at the signal when both laser are on, at $5 \mu \mathrm{s}$, the plasma signal has already grown. If the presence of Rydberg atoms is making the present plasma more bound, we would expect this signal to be similar in size to the Littman laser only signal and shifted to higher field strengths. But the signal with both lasers is larger, suggesting that the Rydberg atoms added by the diode laser are already evolving to plasma. This suggests that the presence of the plasma is accelerating the Rydberg sample to plasma evolution. If we compare the twolaser signal at $15 \mu$ s to the diode laser only signal at $25 \mu$ s, we see that they are similar. This further suggests that the Littman created plasma is having the effect of accelerating the Rydberg to plasma evolution process when both lasers are on. Looking at the two-color signal at $35 \mu$ s we see that the plasma that emerges from the two-laser experiment is still sustained. We see that at the same delay, the plasma that results from using the diode laser only is starting to vanish. Comparing these plasmas suggests that something is happening in the preceding $35 \mu \mathrm{s}$ that is sustaining the two-color plasma at longer delays. The two-color plasma is more robust at longer delays. 


\section{Chapter 4}

\section{Summary and Conclusions}

This thesis has outlined the main threads of ultra-cold plasma research. We have investigated the evolution of an ultra-cold plasma created by photoionization of a sample of $\mathrm{Rb}$ atoms trapped in a magneto-optical trap. We have also observed the spontaneous evolution of a dense sample of Rydberg atoms to an ultra-cold plasma. The result there suggests that the binding energies of Rydberg atoms can be used to stabilize the expansion of cold plasmas. In our two-color experiment, we believe we see preliminary evidence that suggests that Rydberg atoms embedded in an ultra-cold plasma do in fact stabilize and cool the plasma. At this point a qualitative characterization of the plasma electron temperature is needed to verify exactly the effect Rydberg atoms have on the plasma evolution. At this juncture we have yet to pursue such an assessment.

We are pursuing further work to improve the Rydberg atom density. This may enable us to better observe the density dependence of the plasma dynamics. Also, to better probe the intermediary between the cooling and heating regimes, we will use other Rydberg states, and hopefully gain an understanding of the $n$-dependence. Finally, changing the delay between the plasma creation and the 
Rydberg excitation may lead to a more distinct observation of the Rydberg atom influence on the plasma. But control of the delay in this fashion will require a second laser, separate from the current Nd:YAG, incorporated into the timing sequence of the experiment. 


\section{Bibliography}

[1] Wenhui Li, Michael W. Noel, Michael R. Robinson, Paul Tanner, Thomas Gallagher, Daniel Comparat, Bruno Laburthe Tolra, Nicolas Vanhaecke. Thibault Vogt, Nassim Zahzam, Pierre Pillet, Duncan Tate. Phys. Rev. A 70, $042713(2004)$

[2] Nicolas Vanhaecke, Daniel Comparat, Duncan Tate, Pierre Pillet. Phys. Rev. A 71, 013416 (2007)

[3] C.E. Simien, Y.C. Chen, P. Gupta, S. Laha, Y.N. Matinez, P.G. Mickelson, S.B. Nagel, and T.C. Killian. Phys. Rev. Lett. 92, 143001-1 (2004)

[4] Chris Greene, A.S. Dickinson, H.R. Sadeghpour. Phys. Rev. Lett. 85, 2458 (2000)

[5] Thomas Gallagher. Rydberg Atoms. Cambridge University Press, 1994

[6] T. Pohl, T. Pattard, J.M. Rost. Phys. Rev. A 68, 010703(R) (2003)

[7] Wenhui Li, Paul Tanner, T.F. Gallagher. Phys. Rev. Lett. 94, 173001 (2005)

[8] Yukap Hahn. Physics Letters A 293, 266 (2002)

[9] T. Pohl, T. Pattard, J.M. Rost. Phys. Rev. Lett. 92, 155003-1 (2004) 
[10] T.C. Killian, V.S. Ashoka, P. Gupta, S. Laha, S.B. Nagel, C.E. Simien, S. Kullin, S.L. Rolston, S.D. Bergeson. J. Phys. A: Math. Gen. 36, 6077 (2003)

[11] H. J. Metcalf, P. van der Straten. Laser Cooling and Trapping. New York: Springer, 1999

[12] D. E. Pritchard. Phys. Rev. Lett. 51, 1336 (1983)

[13] A. Migdall, J. Prodan, W. Phillips. Phys. Rev. Lett. 54, 2596 (1985)

[14] J.G. Harris, W.C. Campbell, D. Egrov, S.E. Maxwell, R.A. Michniak, S. V. Nguyen, L. D. van Buuren, J.M. Doyle. Review of Scientific Instruments 75(1): 13-24 (2004)

[15] E.L Raab, M. Prentiss, Alex Cable, Steven Chu, D. E. Pritchard. Phys. Rev. Lett. 59, 2631 (1987)

[16] J.M. Raimond, G. Vitrant, S. Haroche. J. Phys.B: At. Mol. Phys. 14, L655 (1981)

[17] M.D. Lukin, M. Fleischhauer, R. Cote, L.M. Duan, D. Jaksch, J.I. Cirac, P. Zoller. Phys. Rev. Lett. 87, 037901-1 (2001)

[18] K. Afrousheh, P. Bohlouli-Zanjani, D. Vagale, A. Mugford, M. Fedorov, J. D. D. Martin. Phys. Rev. Lett. 93, 233001 (2004)

[19] D. Jaksch, J. I. Cirac, P. Zoller, S. L. Rolston, R. Ct and M. D. Lukin. Phys. Rev. Lett. 85, 2208 (2000)

[20] Jan Krueger. http://en.wikipedia.org/wiki/Image:MOT_setup.png 
[21] Jan Max Walter Krueger. A Novel Technique for Frequency Stabilising Laser Diodes. A Thesis, supervisor: Andrew Wilson. University of Otago, October 1998

[22] M.P. Robinson, B. Laburthe Tolra, Michael W. Noel, T.F. Gallagher, P. Pillet. Phys. Rev. Lett 85, 4466 (2000)

[23] W. Li, P.J. Tanner, Y. Jamil, and T.F. Gallagher. Eur. Phys. J. D 40, $27(2006)$

[24] W.P. Spencer, A.G. Vaidyanathan, and D. Kleppner. Phys. Rev. A 26, 1490 (1982)

[25] J.L. Roberts, C.D. Fertig, M.J. Lim, and S.L. Rolston. Phys. Rev. Lett. 92, $253003(2004)$

[26] M. Zheng. Data Acquistion Software Manual for Experiments of Ultra-cold Rydberg Rubidium Atoms. Physics Honors Thesis, Colby College, 2006 